\title{
Der kulturelle Wert historischer Gärten als Geschichtsdokument und Kunstwerk
}

In:

Reinhard F. Hüttl / Karen David / Bernd Uwe Schneider (Hrsg.): Historische Gärten und Klimawandel : eine Aufgabe für Gartendenkmalpflege, Wissenschaft und Gesellschaft ISBN: 978-3-11-060748-2. - Berlin/Boston: De Gruyter Akademie Forschung, 2019 (Forschungsberichte / Interdisziplinäre Arbeitsgruppen der Berlin-Brandenburgischen Akademie der Wissenschaften ; 42)

S. $6-30$ 


\title{
DER KULTURELLE WERT HISTORISCHER GÅRTEN ALS GESCHICHTSDOKUMENT UND KUNSTWERK
}

\begin{abstract}
Eine Analyse des historischen und künstlerischen bzw. kunsthistorischen Wertes einer historischen Gartenanlage muss - insbesondere auch im Hinblick auf sich wandelnde Klima-, Umwelt- und Nutzungsanforderungen - allen Maßnahmen ihrer Pflege, Erhaltung, Wiederherstellung oder Rekonstruktion zugrunde liegen. Sie wird aus den Befundund Quellenuntersuchungen im Abgleich mit den Parametern der Gartenkunstgeschichte und im naturwissenschaftlichen Zugriff (Pflanzenbestand) zielbezogen erstellt (Parkpflegewerk). Aufgrund der lebendigen und fragilen (»natürlichen«) Materialität des Gartenkunstwerks stellen die Sollzustände zwangsläufig Interpretationen und Konstrukte der leitenden Denkmalschicht(en) dar, die ggfs. keinen punktuellen Idealzustand, sondern eine Entwicklung der Anlage veranschaulichen.
\end{abstract}

An analysis of the historic and artistic or art historical value of an historic garden must serve as the basis for all measures taken for its care, conservation, restoration or reconstruction particularly with regard to changing climate, environmental and usage requirements. It involves the targeted examination (park maintenance work) at the in-depth site and sources investigations, and compares them with the parameters for the history of the art of garden design and from the natural science perspective (vegetation). Given the living and fragile (»natural«) materiality of garden artwork, the target conditions necessarily constitute the interpretations and constructs of the most relevant historic garden layer(s) which may not illustrate a sporadic ideal state but rather a development of the site.

Im Text werden Maskulinum und Femininum verwendet, wenn es um Personen geht. Gemeint sind grundsätzlich alle Menschen, gleich welcher Geschlechtsidentität sie sich zugehörig fühlen. 


\section{Der Garten als Geschichtszeugnis}

Wenn von historischen Gärten die Rede ist, geht es um Gärten und Parks, die als Zeugnisse vergangener Epochen oder als Kunstwerke kulturelle Denkmalwerte verkörpern. Der historische Wert von Gartenanlagen ist - den allgemeinen Formulierungen der Denkmalschutzgesetze entsprechend - relativ exakt zu begründen: Oft spielt ihr Stellenwert als Vertreter eines bestimmten Gartentypus die entscheidende Rolle: Von den fürstlichen Residenzgärten der höfischen Gesellschaft reicht das Spektrum über die feudalen Gutsgärten, die städtischen Bürgergärten, die suburbanen Villengärten, die Gärten berühmter Literaten, Denker, bildender Künstler oder sonstiger prominenter Zeitgenossen bis hin zu den kurz vor 1800 aufkommenden kommunalen Volks- oder Stadtparks und Parkfriedhöfen, den modernen Gartenstädten der Lebensreformbewegung um 1900 und den jüngsten Gartenexperimenten der Moderne und Postmoderne. Häufig gibt die historische Bedeutung des Bauherren, Gartenkünstlers, Besitzers oder Nutzers den Ausschlag. Manchmal geht es auch »nur« um den Zeugniswert einzelner Naturelemente (Stritzke 1998). Als Dokumente sind historische Gärten materialisierte Zeugnisse geschichtlicher Zustände, Ereignisse und Ideen (einschließlich religiöser, politischer, sozialer, wirtschaftlicher, biographischer und naturwissenschaftlicher Faktoren und wissenschaftsgeschichtlicher Aspekte). ${ }^{1}$ Sie sind stets einzigartige Vertreter einer klassifizierbaren Gattung und eines Typus, die an Zeit, Ort, Funktion und Akteure gebunden sind und denen aus heutiger Sicht immer aufs Neue historische Bedeutung zugemessen werden muss: sei es im lokalen, im regionalen, nationalen oder gar im globalen Rahmen.

Die historischen Bewertungen sagen zunächst noch nichts über die Beschaffenheit der Gestaltung aus, die zwangsläufig mit der Anlage von Gärten verbunden ist: Selbst reine Nutzgärten waren und sind als kultivierte Ausschnitte aus der »Wildnis« der Natur oder aus dem Betondickicht der Städte an eine Form gebunden, die ursprünglich funktional bedingt war - wie etwa die Quincunx-Pflanzungen der Obstplantagen oder die orthogonalen Beetreihen der mittelalterlichen Kräuter- und Gewürzgärten. Doch erfüllte der Garten darüber hinaus fast immer auch höhere ideelle, mystische, religiöse und repräsentative Funktionen: Das Ineinandergreifen von Nutzen und ästhetisch-symbolischer Ordnung durch mehr oder minder kunstvolle Gestaltung ist in der Entwicklungsgeschichte der Gärten die Regel: Das gilt für die ältesten ägyptischen und babylonischen Anlagen über die antike griechisch-römische Villenkultur und die orientalischen Gartenoasen nach dem Tschahār-Bāgh-Schema ebenso wie für ihre frühneuzeitlichen und barocken Nachfolger bis hin zur expliziten Versöhnung des Schönen mit dem Nützlichen (Burgsdorf 1791, 120) in den Landschaftsgärten des 18. und 19. Jahrhunderts. Es lässt sich festhalten, dass der historische Wert der Gärten von ihrer künstlerischen Gestaltung und kulturellen Bedeu- 
tung kaum zu trennen ist. Das bringt für ihre konkrete Bewertung und für die Strategien ihrer Erhaltung bzw. Wiederherstellung unter den heutigen Nutzungsanforderungen erhebliche methodische Probleme mit sich.

\section{Der Garten als Heterotop und Gartenkunstwerk}

Inwieweit ein historischer Garten als Kunstwerk zu definieren ist und welche Konsequenzen daraus zu ziehen sind, ist oft umstritten. Seine Definition als Artefakt steht jedoch bei aller Naturhaltigkeit außer Frage und lässt sich im Sinne Michel Foucaults am besten als Heterotop verstehen: Heterotope sind nach Foucaults Definition

"wirkliche Orte, wirksame Orte, die in die Einrichtung der Gesellschaft hineingezeichnet sind, sozusagen Gegenplatzierungen oder Widerlager, tatsächlich realisierte Utopien, in denen die wirklichen Plätze innerhalb der Kultur gleichzeitig repräsentiert, bestritten und gewendet sind, gewissermaßen Orte außerhalb aller Orte, wiewohl sie tatsächlich geortet werden können« (Foucault 1967, 39).

Foucaults Kunstgriff - in unserem Falle zur Beschreibung zugleich materieller und illusionärer Gartenwelten - erlaubt uns, historische Gärten auf verschiedenen Ebenen zu verorten und bezüglich der dem Gartenkünstler zur Verfügung stehenden künstlerischen Gestaltungsmittel und Ideenwelten gesondert zu betrachten, gleichsam als eine »dritte Natur«(de Jong 1998).

Dabei müssen wir allerdings den neuzeitlichen Kunstbegriff und seine Anwendung auf den Garten erst einmal in Frage stellen. Versteht man »Kunst « im antiken Sinne als Kunstfertigkeit ( $\tau \dot{x} \chi \nu \eta)$, die ein handwerkliches Erfahrungswissen umfasste, so erforderte die Anlage von Gärten stets vielerlei Kompetenzen und somit auch geteilte Autorschaften ${ }^{2}$ : Unverzichtbar war zu allen Zeiten der Gärtner mit seinen profunden Kenntnissen der Pflanzen, des Wetters, der Erden, der Pflege, Düngung und Aufzucht, also des gesamten Gartenhandwerks. Hinzu kamen die Vermesser, Architekten und Ingenieure für die Eingrenzung, Einteilung und Nivellierung, für die Anlage der Be- und Entwässerung, der Terrassierungen, Treppen und festen Wege, Brunnen und Fontänen, Mauern, Tore und Ziergebäude (Abb. 1) - und schließlich die Bildhauer und Maler für den Bildschmuck. Dahinter stand zumeist ein ordnender Geist, manchmal der Bauherr selbst, der oft als dilettierender Autor auftrat. Im Hinblick auf die höhere Geometrie und Mathematik der Gestaltung sowie auf ihre theologische, mythologische, philosophische, poetische - im weitesten Sinne allegorische - Programmatik wurden im multidisziplinären Gartenkunstwerk die Grenzen zwischen den praktisch-handwerklichen Künsten und den gelehrten und freien Künsten und später auch zu den modernen Naturwissenschaften (etwa zur Botanik) letztlich immer wieder aufgehoben.

2 Vgl. den Beitrag von Christiane Salge in diesem Band. 


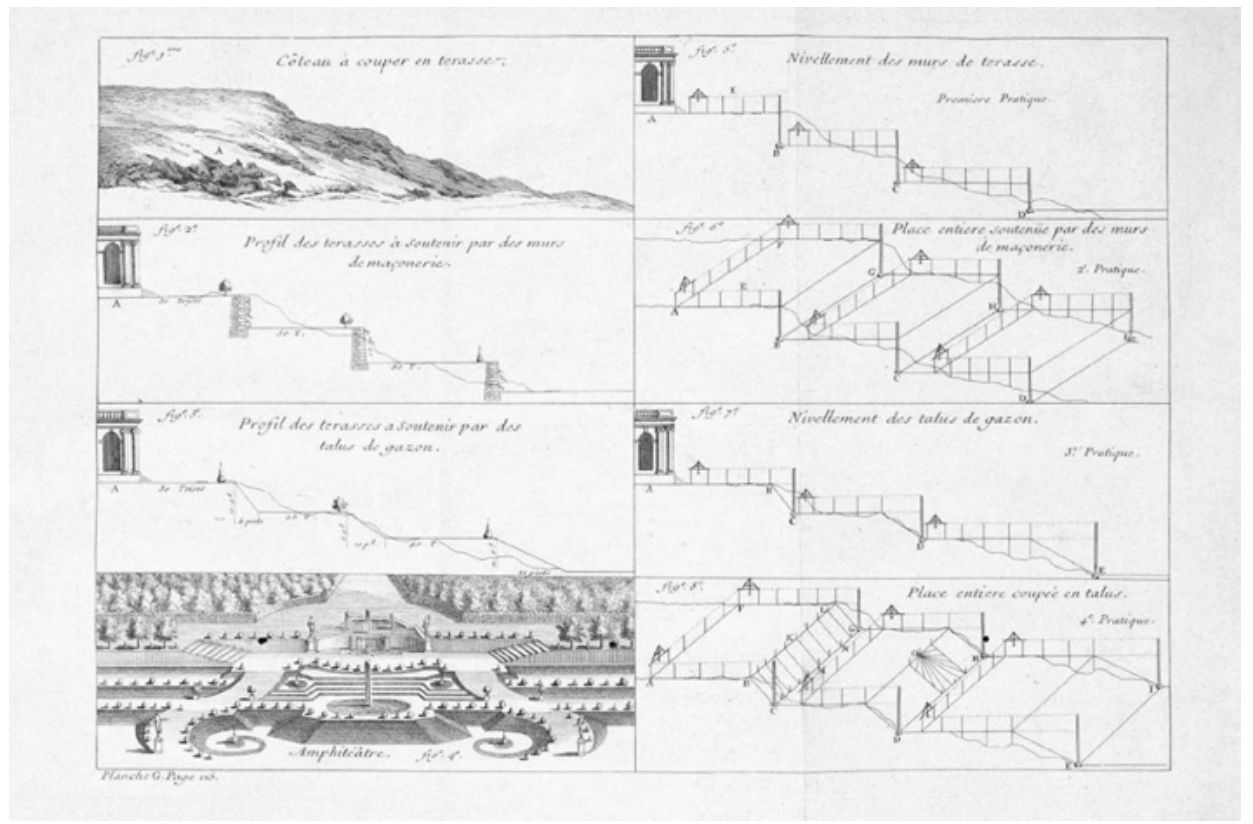

1 Alexandre Le Blonde, Anlage eines terrassierten Barockgartens, Kupferstich, 25,7×38cm. In: Dézailler d’Argenville, A.-J. (1709): La théorie et la pratique du jardinage. Paris: Jean Mariette, 116, 1-8.

Die künstlerische Dimension historischer Gärten lässt sich nur im jeweiligen Spannungsfeld der Künste, Handwerke und Wissenschaften beschreiben und bewerten. Man hat deshalb sogar von der »Erfindung der Gartenkunst « (Fitzner 2012; Schweizer 2013) seit dem 16. Jahrhundert gesprochen, weil es tatsächlich nie wirklich gelang, die Gartenkunst als solche zu einer autonomen künstlerischen Disziplin und damit zu einem Lehrfach an den Universitäten und Akademien zu erheben. Der Gartentheoretiker Christian Cay Lorenz Hirschfeld, dem wir nach Horace Walpole (Walpole 1770/1904) die erste umfassende Geschichte und Theorie der Gartenkunst verdanken (Hirschfeld 1779-1785), hatte zwar einen Lehrstuhl an der Christian-Albrechts-Universität zu Kiel inne, der aber war ganz allgemein den »Schönen Künsten « gewidmet (Schepers 1980; Kehn 1992). Auch die von Minister von Altenstein und Peter Joseph Lenné 1823/24 gegründete preußische Gärtnerlehranstalt in Potsdam mit den Qualifikationszielen Gärtner, Kunstgärtner, Gartenkünstler konnte solche hohen Doppelerwartungen an exakte Wissenschaftlichkeit und universales Künstlertum letztlich kaum erfüllen (Wimmer 2016, 119-134), und die Einrichtung von Professuren für Gartenarchitektur, Grünplanung, Gartenforschung und Gartendenkmalpflege im Laufe des 20. Jahrhunderts verstand sich folgerichtig schon jenseits eines explizit definierten gartenkünstlerischen Anspruchs. 


\section{Die Deutungshoheit der Kunsthistoriker}

Blickt man auf die Geschichte der historischen Gartenforschung zurück (Wimmer 1989; Buttlar 2003; 2004), so zeigt sich, dass die Autorschaft und Deutungshoheit von den Architekten, Ingenieuren und universal gelehrten Hofgärtnern, Dilettanten und Literaten des 18. Jahrhunderts zunächst auf die professionelle Zunft der Landschafts- und Gartenarchitekten überging (Humphry Repton, John Claudius Loudon, hierzulande z.B. Friedrich Ludwig von Sckell, Peter Joseph Lenné und Gustav Meyer, Hermann Fürst von PücklerMuskau). Erst seit im letzten Drittel des 19. Jahrhunderts die junge akademische Fachdisziplin der Kunstgeschichte Gärten als Kunstwerke sui generis einzuschätzen begann, wurde der kunsthistorische Stilbegriff auf deren Gestaltung angewendet, wobei man sich eine lineare Entwicklung vom architektonisch-geometrischen Barockgarten zum vermeintlich »natürlichen « Landschaftsgarten vorstellte (Gothein 1914; Hallbaum 1927). Auf einer Zeitschiene wäre demnach jeder Garten eindeutig zu verorten. Wir wissen heute zwar, dass dieses Modell viel zu eindimensional und mechanisch gedacht ist, aber dennoch lässt sich in der Regel der Wandel der künstlerischen Repräsentation von Natur anhand historischer Bildquellen und originaler Befunde im Gelände ablesen und stilvergleichend bewerten.

Die zweite Übertragung aus dem Fach Kunstgeschichte war die im Kontext der Mittelalter-und Renaissanceforschung entstandene Ikonographie und Ikonologie: die Auswertung verifizierbarer Hinweise auf inhaltliche Aussagen, Bedeutungen und Identifikationen, die sich im Rahmen multidisziplinärer Fragestellungen heute mit der Erforschung des kulturgeschichtlichen Kontextes der Gärten verbinden.

Die bewährte Expertise zur Entzifferung von Plan- und Bildquellen, Allegorien, Emblemen und Symbolen sowie die Zuordnung von Formen und ästhetischen Befunden zu historischen Fakten, philosophischen, theologischen und literarischen Systemen und Texten prädestiniert das Fach Kunstgeschichte, auch historische Gärten in komplexer Weise $\mathrm{zu}$ »lesen « - allerdings im Bewusstsein der besonderen hermeneutischen Fallen, die das Gartenkunstwerk bereithält: Dazu gehört in erster Linie sein fragiler Werkcharakter.

\section{Fragilität und Essenz des Gartenkunstwerks}

Im Gegensatz zu den herkömmlichen Kunstgattungen der Architektur und Bildnerei fehlt dem Gartenkunstwerk, soweit es aus natürlichen und lebendigen Materialien besteht, das wichtigste Kriterium von Monumentalität: die die Zeit überwindende Dauer. Nur seine architektonischen und skulpturalen Elemente überleben längerfristig, alles andere kann dem Gesetz der Entropie nur entkommen, indem es sorgsam gepflegt, d.h. immer wieder aufs Neue hergestellt wird: Hecken und Bäume müssen beschnitten, Haine gelichtet, Wege befestigt, Rasen gewalzt und die Zu- und Ableitungen der Gewässer gereinigt, Teiche entschlammt und Parterres jährlich neu bepflanzt werden, um den Status quo zu erhalten. Darin liegt ein enormer Erhaltungsaufwand (der auch heute unter gartendenkmalpflegeri- 
schen Gesichtspunkten ein Luxus bleibt). Nicht zuletzt entstehen Gärten zumeist über Jahre hinweg, bevor sie ihren Sollzustand erreichen, auch wenn Ludwig XIV. die Parterres seines Grand Trianon in Versailles über Nacht auswechseln ließ und Fürst Pückler mit aufwendigen Methoden ausgewachsene Bäume anpflanzte (allein in Branitz etwa 700), um den Reifungsprozess seiner Anlagen abzukürzen (Wecke 2016). Andererseits wachsen Bäume oft unkalkuliert aus und verändern die Maßstäblichkeit und Wirkung der Gartenszenen, etwa durch optische Verkleinerung von Gartentempeln, Wasserfällen oder Hügeln, durch Verschattung der Gewässer, Wege und Lichtungen und durch Überwuchern der Sichtachsen. Hinzu kommt, dass auch aus anderen Gründen die Halbwertszeit historischer Gärten selten länger als ein bis zwei Generationen betrug: Fast mit jedem Besitzerwechsel, jedem Wandel des Geschmacks und der Mode (taste, fashion), der Repräsentationsfunktion und gesellschaftlichen Nutzung sind Veränderungen verbunden. Pückler zweifelte sogar daran, überhaupt »in der landschaftlichen Gartenkunst ein bleibendes, fest abgeschlossenes Werk liefern« zu können (Lauterbach 2016, 53).

Dennoch gilt es, aus der Analyse des Bestandes und der Quellen die wichtigste Epoche und künstlerische Schicht (das ist oftmals nicht die älteste) oder auch die bedeutendste Abfolge mehrerer historischer Denkmalschichten idealtypisch herauszuarbeiten und pflegend zu bewahren oder wieder herzustellen. Insofern ist die Gartenkunstgeschichte im Hinblick auf partielle Rekonstruktionen zwangsläufig toleranter als die Baudenkmalpflege (Buttlar 2008; 2012). Die heutige Gartenforschung ist methodisch soweit fortgeschritten, dass sie in Form von Parkpflegewerken in der Regel die historischen Befunde und Schichten der Anlagen so genau erfassen und definieren kann, dass daraus handhabbare Leitlinien zur Sanierung, Pflege und Rekonstruktion resultieren (Karg 1989; Rohde/Schomann 2003, 246-295; Meyer o.D.). ${ }^{3}$ Diese Leitlinien markieren klare Grenzen gegen zerstörerische Eingriffe in die künstlerische Essenz des historischen Gartens. Die historische und kunsthistorische Bewertung historischer Gärten muss somit die Basis für alle weiteren Entscheidungen über ökologische Fragen wie Natur- und Artenschutz sowie soziale und touristische Nutzungswünsche sein - Kriterien, die erst unter Berücksichtigung der vorrangigen kulturellen Erhaltungsziele in das interdisziplinär aufgebaute Parkpflegewerk einfließen.

\section{Künstlerische Aspekte}

Als Gesamtkunstwerke können die barocken Gärten mit Hilfe ihrer wirkmächtigen formalen Gestaltung und ikonografischen Ausstattungsprogramme (z.B. Dezallier d’Argenville 1709/1760; Finckenstaedt 1966; Erichsen-Firle 1971; Dennerlein 1981; Lauterbach 1987; Brix 2004; 2009) komplexe symbolische und allegorische Aussagen repräsentieren, etwa zur Repräsentation gesellschaftlicher und höfischer Ordnung (Krause 1996), zu den Topoi des 
verlorenen (und wiedergefundenen irdischen) Paradieses, zum mythischen Arkadien und Elysium, zur Macht Gottes, der Götter und des Herrschers, zu dessen historisch-dynastischer Legitimation oder auch zum Lebens- und Erkenntnisweg des Menschen, zum Spannungsfeld zwischen Stadt und Land, Macht und Moral, Wildnis und Zivilisation, Natur und Kunst, Geschichte und Poesie. Solche inhaltlichen Dimensionen historischer Gartenkunstwerke zu ergründen, erweist sich oft als noch schwieriger als die Bewertung ihrer formalen Strukturen (Buttlar 1981; 1982, 95-166; 2007; Stempel 1982; Schweizer/Winter 2012, 253-352). Denn Vieles ist nur fragmentarisch überliefert, und im Laufe der Zeit muss mit Veränderungen und Störungen der Zusammenhänge - beispielsweise von Skulpturenprogrammen - gerechnet werden. Dem kann hier im Einzelnen nicht nachgegangen werden. Nur so viel:

Gärten als begehbare Kunstwerke besitzen stets eine künstlerisch ausgestaltete, auf den menschlichen Leib und die Eigenbewegung (Kinästhesie) bezogene Topographie: ein Links und Rechts, Vorn und Hinten, Oben und Unten, Vorher und Nachher. Die anschaulich beschreibbare Topographie wird zur Topologie, wenn die vielen, durch Geländemodellierung, Bauten, Statuen, Grotten, Inschriften ausgezeichneten und über Sichtachsen, Wege und Treppen miteinander verbundenen »Orte« einen programmatischen Sinnzusammenhang (Ikonologie) erkennen lassen. Nicht zuletzt sind bei der historischen Pflanzenverwendung neben dem Artenschutz der tradierte Symbolgehalt und - im sentimentalen Garten des 18. Jahrhunderts - der Stimmungswert der Pflanzen zu berücksichtigen (Nath-Esser 1990; Holmes 2006; Wimmer 2014). Für konrete ikonologische Gartenanalysen gibt es mittlerweile einige aufschlussreiche, wenngleich nicht unumstrittene Beispiele (Wiener Belvedere, Villa d'Este, Bomarzo, Pratolino, Hortus Palatinus Heidelberg, Kassel-Wilhelmshöhe, Schwetzingen, Stowe, Stourhead). ${ }^{4}$ In unserem Untersuchungsbereich wären beispielsweise Park Sanssouci und der Neue Garten in Potsdam zu nennen (Buttlar/Köhler 2012; Ruge 2013).

Die schon von Hirschfeld 1779 als solche bezeichnete »Gartenrevolution « des 18. Jahrhunderts (Hirschfeld 1779-1785, Bd. I, 121) stellte nicht nur eine ästhetische Kritik des formalen Gartenstils dar, sondern signalisierte im Zuge der Aufklärung eine tiefgreifende Veränderung des gesellschaftlichen und ethischen Verhältnisses von Mensch und Natur. Der freien Natur wurde im Landschaftsgarten nun im Geiste der Physikotheologie etwa Isaac Newtons und des moralisierenden Deismus Lord Shaftesburys sogar die Rolle einer höchsten göttlichen Instanz zugesprochen (Shaftesbury 1709, in: Hunt/Willis 1975, 122-124). Wo Freiheit aus dem Naturrecht begründet wurde - wie im fortschrittlichen England und im vorrevolutionären Frankreich -, konnte umkehrt unverfälschte Naturform zum Freiheitssymbol werden (Gerndt 1981; Buttlar 1982, 140-145; Gamper 1998). Der gärtnerische Beschnitt der Pflanzen wurde in diesen Diskursen von nun an als Vergewaltigung der

4 Aurenhammer 1956; Coffin 1960; Woodbridge 1965; 1970 (dazu kritisch: Schäfer-Stöckert 2018); Clarke 1973; Patterson 1981; 1982; Bredekamp 1984; Vezzosi 1985; Internationales Symposium des Deutschen Nationalkomitees von ICOMOS, der Museumslandschaft Hessen Kassel und des Landesamtes für Denkmalpflege Hessen 2010; Hesse 2012; Niedermeier 2012. 
Natur empfunden, während der freiwachsende Baum den aufgeschlossenen Zeitgenossen »edler als ein Monarch im Krönungsornat « erscheinen konnte - so der Dichter und Propagandist der neuen Gartenkunst, Alexander Pope (nach: Osborn 1966, No. 616). Aus der Auflösung der strengen Ordnung der Barock- und Rokokogärten entwickelte sich im zweiten Drittel des 18. Jahrhunderts auf den englischen Landsitzen und Villen der aufsteigenden liberalen Elite - neuadelige Grundbesitzer, bürgerliche Dichter, Politiker, Banker, Handelsmagnaten - schrittweise der englische Landsitz als Symbol eines liberalen Weltentwurfs (Buttlar 1982). Im letzten Drittel des Jahrhunderts trat der Landschaftsgarten seinen Siegeszug auf dem Kontinent an, wobei er namentlich von den aufgeklärten »kleineren Prinzen« und Adeligen Deutschlands (Walpole 1770/1904, 89) rezipiert und hinsichtlich seiner Form und Botschaft den kleinteiligen politischen und differenten gesellschaftlichen Verhältnissen hierzulande angepasst wurde (Buttlar 1981; Tabarasi 2007; Siegmund 2011).

Als Kunstform ist der Landschaftsgarten mit seiner Forderung, die Schönheiten der Natur nachzuahmen und dabei sogar ins Ideale zu steigern (improvement), nicht leicht zu definieren: Der Vorschlag, sich am Vorbild der aus meditativer Intuition gestaltenden chinesischen Gartenkunst (Sharawadgi, als Kunst der schönen Unregelmäßigkeit) zu orientieren, scheiterte, weil - wie Sir William Temple schon 1692 formulierte - »die Mentalität der Chinesen von unserer europäischen so weit entfernt ist wie ihr Land « (Temple, zit. n. Hunt/ Willis 1979, 99). Stattdessen setzte sich ab den 1720er Jahren eine szenische, vom Theater beeinflusste Gestaltung durch (Lang 1974): Wie auf einer Bühne wurden nun Baumgruppen, Gewässer und Kleinbauten (klassische Tempel, Brücken, Pyramiden, Obelisken, Gedenksäulen, Ruinen, Eremitagen etc.) als Naturszenen im irregulären Netzwerk von Wegen und Plätzen inszeniert. Die gerade Linie und die letzten geometrisch geformten Kanäle und Bassins wurden bald durch Schlängelwege und serpentinenförmige Teiche, die beschnittenen Hecken und Bäume durch frei wachsende Pflanzungen ersetzt. Die neue »natürliche« Gestaltungsweise müsse jedoch - so forderte Alexander Pope 1731 - stets den bereits im Terrain vorgegebenen Möglichkeiten, dem individuellen Genius of the Place (Pope 1731, Vers 57ff.) folgen. Etwas später sprach der professionelle Landschaftsgärtner Lancelot Brown von den spezifischen Chancen - den capabilities - eines Parkgrundstücks als Ausgangspunkt jeder Gestaltung (weshalb er den Spitznamen Capability Brown erhielt).

Einfühlung in den genius loci allein aber reichte nicht aus. Wo Natur zwar als wichtigstes Material dienen, zugleich aber auch als Medium einer künstlerischen Aussage über sich selbst hinausweisen sollte, bedurfte es neuer künstlerischer Regeln. Infolgedessen wurde die englische Gartenkunst in ihrer zweiten Phase um die Mitte des 18. Jahrhunderts an den Kompositions-und Gestaltungsprinzipien der Landschaftsmalerei orientiert (verschiedene, ineinander verschränkte Bildgründe, attraktive Architekturmotive in »malerischer « Schrägsicht, Repoussoirs im Vordergrund, belebende Farbperspektiven und Licht-SchattenEffekte). Der Landschaftsgarten stellt idealisierte Natur in dreidimensionalen, begehbaren Bildern dar - er wurde immer wieder mit einer virtuellen Bildergalerie verglichen (Spence 1752, zit. n. Osborn, No. 1134; Pückler 1834, 36). Dies setzte freilich optimale Betrachterstandpunkte voraus, die auf dem Gartenrundgang oft durch Inschriften oder Ruhebänke 
markiert und in den gedruckten Gartenführern erläutert wurden. Als Vorbilder galten die mit antiken Versatzstücken ausstaffierten Ideallandschaften Claude Lorrains und Gaspard Poussins, die noch an arkadische Mythen oder pastorale Poesie anknüpften. Mit den Augen Claudes in den heimischen Gärten manch neues Tivoli zu schaffen, forderte der englische Dichter William Mason 1772 in seinem Lehrgedicht The English Garden (Mason 1772/1785, 4 Verse, 63ff.). Für wildere »nordische« Szenen(z. B. Felsengebirge, Wasserfälle, Schluchten) boten sich neben Salvatore Rosa die gleichermaßen berühmten niederländischen Landschaftsmaler Jakob van Ruysdael und Allaert van Everdingen an. Gezielt entstanden auf diese Weise sogar in ein und demselben Garten bewusst inszenierte Gegenbilder, die im Kontrast zur klassischen Antike an die glorreichen Ursprünge der nordischen Kulturen und Nationen erinnerten (Landeshauptstadt Stuttgart 1994; Buttlar 2001; Weyh 2006; Niedermeier 2007b).

Der ikonologische Zusammenhang des zunehmend deutungsoffener werdenden Gartenkunstwerks lässt sich heute (soweit keine expliziten Quellenaussagen vorliegen) nur noch nach Maßgabe zeittypischer Rezeptionsweisen eines imaginierten historischen Betrachters, seines Bildungshorizontes, seiner Gefühlswelt und potentieller Assoziationsketten erschließen: Dafür spricht, dass parallel zur Gartenentwicklung die neuen Wahrnehmungstheorien der Imagination, des Sensualismus und der Assoziation entstanden (Addison 1712; Burke 1757; Alison 1790). Die Gestaltung wurde dementsprechend zunehmend von der Nachahmung kunstgeschichtlicher Bildvorlagen und dem zugehörigen Bildungswissen losgelöst und unmittelbarer auf die sinnliche Wahrnehmung und das erwünschte Spektrum der durch Form und Farbe ausgelösten Stimmungen, Empfindungen und Assoziationen bezogen (Whately 1770). Der Charakter der Szenen, beispielsweise des locus melancholicus (Abb. 2), ist im sentimentalen Garten nicht zuletzt durch den katalogartig aufgelisteten Ausdruckswert der verwendeten Bäume bestimmt (Hirschfeld 1779-1785, Bd. IV, 82; Buttlar 2019a). ${ }^{5}$ Dies gilt auch für die Wege als stumme Führer des Spaziergängers (Verschragen 2001). Friedrich Ludwig von Sckell zeigt 1818, wie der Gärtner, sich seiner Intuition überlassend und unwillkürlich der sogenannten Schönheitslinie (Hogarth 1753) folgend, das Gelände durchschreitet und dabei rückwärtig mit einem langen Stab den zukünftigen Spazierweg markiert (Sckell 1825, 76) (Abb. 3).

Gartenästhetik und Gartentheorie erreichen im breit geführten Diskurs des späten 18. Jahrhunderts (u.a. Shenstone 1764; Whately 1770; Chambers 1772; Hirschfeld 17791785; Gilpin1786; 1792; Girardin 1788; Repton 1795; 1803; 1816) - man denke etwa auch an Goethes Gartenroman Die Wahlverwandtschaften (1809) - einen ungemein hohen Grad der Differenzierung ihrer künstlerischen Mittel, der auch rückblickend in der aktuellen Bewertung des Gartendenkmals berücksichtigt werden muss: von der Geländemodellierung $^{6}$ und Raumbildung über die Regie der malerischen Gewässer, die historische Pflan-

5 Vgl. den Beitrag von Florian Abe in diesem Band.

6 Vgl. den Beitrag von Karen David in diesem Band. 


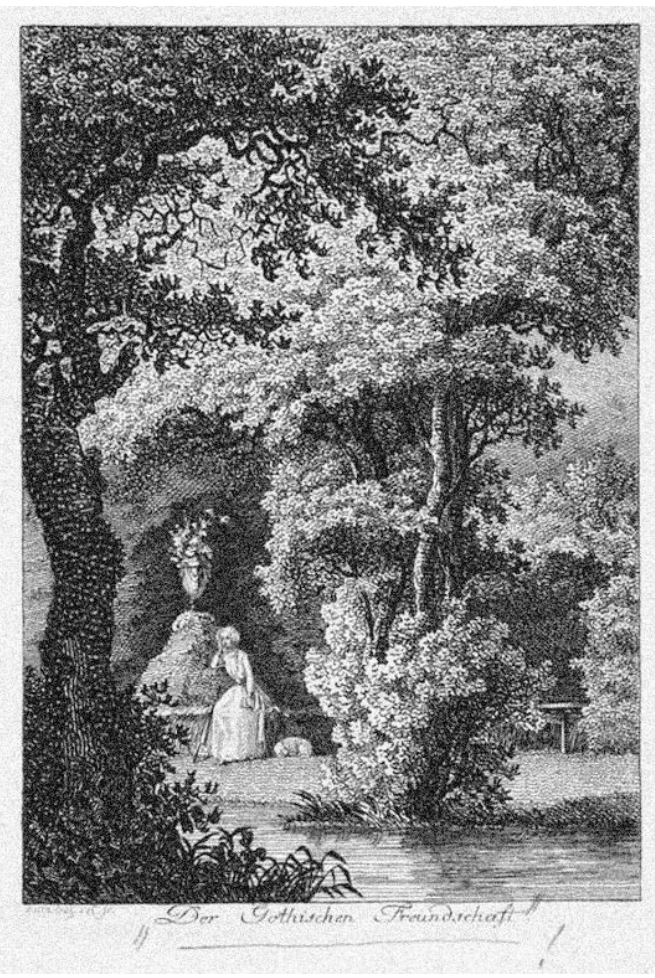

2 J.A. Darnstedt, Melancholische Szene im Landschaftsgarten, Kupferstich. In: Becker, Wilhelm Gottlieb (1792): Das Seifersdorfer Thal. Leipzig: Voß und Leo.

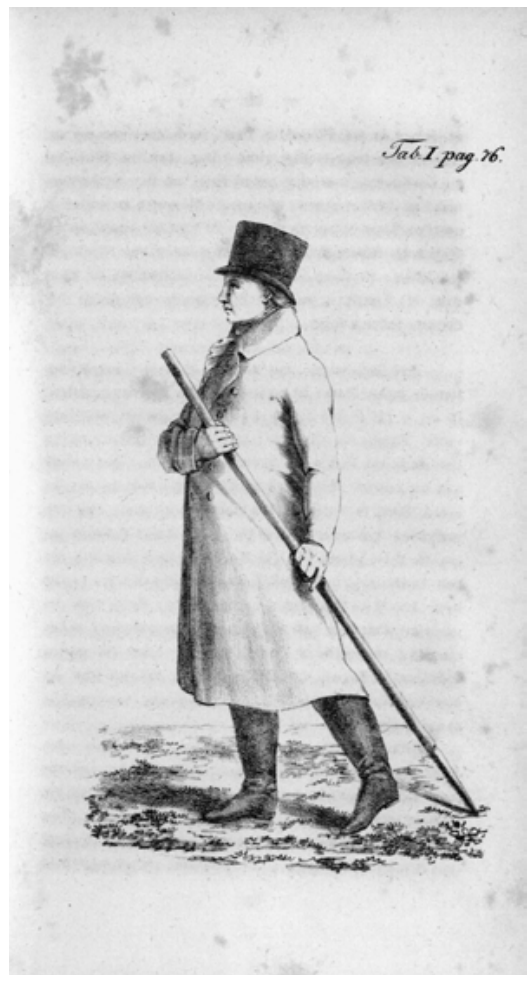

3 Der Gartenkünstler beim Anlegen eines Weges. In: Sckell, Friedrich Ludwig von $\left(1825^{2}\right)$ : Beitraege zur bildenden Gartenkunst für angehende Gartenkünstler und Gartenliebhaber. München: Lindauer.

zenverwendung, das breite Spektrum der schmückenden Staffagen als Stimmungsträger bis zu dem Farbenrausch der Baum- und Buschpflanzungen, wie ihn Eduard Petzold reflektierte (Rohde 1998).

\section{Kurze Charakterisierung der vier ausgewählten Mustergärten unter den Kriterien ihrer historischen und künstlerischen Bedeutung}

Wörlitz: Der Schlossgarten zu Wörlitz ist dem substantiellen Bestand nach der älteste der vier Mustergärten - Zentrum des sogenannten Wörlitzer Gartenreiches des aufgeklärten Fürsten Leopold Friedrich Franz von Anhalt Dessau (1740-1817). In der Partie rund um das von Friedrich Wilhelm von Erdmannsdorff ab 1763 im neopalladianischen Stil an Stelle eines älteren Jagdschlosses errichtete Englische Landhaus sind die ältesten, noch die 
barocken Seitenalleen aufnehmenden Gestaltungen erhalten (Trauzettel 1996; 2006). Jenseits des aus einem Elbaltwasser entstandenen Sees (Resultat des genius loci) hat sich die Anlage sukzessive nach der ersten großen Überschwemmung 1772 weiterentwickelt: Neumarks Garten mit dem kleinteiligen Labyrinth, Schochs Garten um das Gotische Haus mit der dahinterliegenden mystischen Partie sowie die ab den 1790er Jahren entstandenen Neuen Anlagen im Osten zwischen dem Pantheon und der Insel Stein im Süden, einer reich mit Reiseerinnerungen an den Golf von Neapel bestückten Nachbildung des Vesuv (Rode 1814). Der herausragende künstlerische Wert des Gartens resultiert vor allem aus dem einzigartigen Netzwerk der verdoppelten Wegeführung zu Land und zu Wasser. So erschließt sich dem Parkwanderer ein komplexes Spektrum sich überschneidender Sichtachsen und ineinander gleitender, immer wieder neuer und anderer Gartenbilder. Der vorbildlichen, schon in der DDR-Zeit praktizierten Gartendenkmalpflege unter Hans Hallervorden, Kurt Lein und Ludwig Trauzettel ist es zu verdanken, dass dieses zwischenzeitlich stark verwilderte und zugewachsene System der ästhetischen und geistigen Konnektivität heute im Geiste des 18. Jahrhunderts wieder erlebbar ist (Trauzettel 2003; Fibich 2013).

Wörlitz schließt als erstes und prominentestes Beispiel der Rezeption an den Status der Landschaftskunst in England an, die Fürst Franz, der Architekt Erdmannsdorff und der Gärtner Eyserbeck auf ihren wiederholten Gartenreisen nach England ab1763/64 (Weiss 1997) sehr genau studierten. ${ }^{7}$ Die verschiedenen Bereiche lassen in der Wegeführung und Bepflanzung die stilistische Entwicklung von der kleinteiligen irregulären Manier (Neumarks Garten) zum großräumigen, mit Baumgruppen (clumps), geschlossenen Konturen, gürtelartigen Kulissenpflanzungen (belt) und weiten hellen Lichtungen und Wasserflächen arbeitenden klassischen Stil der Zeit um 1800 (Hallbaum 1927) erkennen, etwa in der Partie des Großen Wallochs. Die zeitgenössische Bildpropaganda, die die Wörlitzer Anlagen über Serien von Stichen und Aquatintablättern der Chalkographischen Gesellschaft Dessau an die Gartentouristen Europas vermittelte, liefert der Gartendenkmalpflege heute wertvolle Aufschlüsse, wie die Gartenbilder gesehen werden sollten. Das schließt etwa auch die auf den ersten Blick irritierende Nachpflanzung von nordischen Koniferen vor der Garten-

7 So entspricht das Schloss (obwohl in Details der archäologischen Antikenrezeption darüber hinausgehend) einem in England bereits etablierten neopalladianischen Typus, der »Englische Sitz und das drei Jahrzehnte später entstandene Pantheon sind ohne Kenntnis von Stourhead/Wiltshire nicht zu denken, während das Gotische Haus sowohl auf den »Tempel der Freiheit« in Stowe als auch auf Horace Walpoles Strawberry Hill in Twickenham zurückgeht (und dies, obwohl die originelle Nordfassade von einer venezianischen Kirche und die jüngere Ostfassade vom Breslauer Rathaus inspiriert ist). Der Venustempel zeigt, übrigens auch in seiner programmatischen Aussage zu Libertinage und Tugend, enge Verwandtschaft mit Lord Dashwoods Venusheiligtum in West-Wycombe/Buckinghamshire; die sogenannte Chinesische Brücke geht ebenso wie der künstliche Vulkan des »Steins « auf die Traktate von William Chambers zurück, die Grottengänge im Labyrinth erinnern an Alexander Popes bekanntes Souterrain seiner TwickenhamVilla, und die Ädikulen mit den Büsten des gerade verstorbenen Dichters Johann Fürchtegott Gellert und des damals noch lebenden Schweizer Physiognomen und Freundes Johann Caspar Lavater sind dem Schrein der »British Worthies« in Stowe verblüffend ähnlich. Die Rousseau-Insel hingegen verweist als direktes und zeitnahes Zitat auf das Grab des großen Philosophen, den das Fürstenpaar einst auf einer Parisreise besucht hatte, im Landschaftsgarten von Ermenonville. 
fassade des Schlosses ein, die wohl bewusst mit der Italianità der Architektur kontrastieren sollten. Pflanzenikonographische Botschaften sind zudem mit dem Kindergrab der Goldenen Urne (Rotzedern und Pappeln als Ersatz für Zypressen - den antiken mediterranen Trauerbäumen) und der gleichfalls mit Pappeln bepflanzten Rousseau-Insel verbunden. Der üppige und ornamental präsentierte Blumenschmuck am Floratempel einschließlich des rekonstruierten Phallusbeetes beschwört hingegen den Priapus-Kult und die Raffinesse antiker Villengärten (Niedermeier 1995, 191-228), wie sie von Humphry Repton ab den 1790er Jahren als in den Landschaftsgarten reintegrierte formale Gestaltungselemente (Separatgärten) propagiert wurden. Was die Auswahl der Ein- und Ausländischen Baumarten in Wörlitz anbetrifft, so liefert das Verzeichnis der Bäume, Sträucher, Pflanzen und Stauden des jüngeren Schoch mit 462 Gehölzarten und 644 weiteren Spezies genauere Aufschlüsse (Rode 1814, 195-240).

Besonderes Augenmerk verdient der Wörlitzer Park darüber hinaus in zweierlei Hinsicht: Zum einen versteht er sich auch als eine experimentelle Zierfarm (ferme ornée), deren diverse Obstquartiere mit sorgsam gezüchteten Fruchtsorten ebenso integriert waren wie Schaf- und Viehweiden, Felder und Äcker (mit Saatgutexperimenten) oder auch forstästhetische Ansätze wie die Aufpflanzung des Sieglitzer Berges (Lott 1994). Zum anderen ist der Schlosspark in die großräumige Elbauenlandschaft mit ihren Schutzdeichen und mit uralten Eichen besetzten Huden so eingebettet, dass nicht nur die Gartenbilder im Inneren des Parks, sondern auch die Fernblicke nach außen auf die Schmuckarchitekturen der Wallwachhäuser (die wiederum zugleich überlebenswichtige Nutzbauten zum Hochwasserschutz waren $)^{8}$ ihn in der Dessauer Landesverschönerung (Friedrich von Matthison) aufgehen lassen. Eine solche Grenzüberschreitung ins Utopische lässt sich analog für den ideellen Gehalt dieser einzigartigen Kunstlandschaft nachweisen (Hirsch 1985; 1995; Niedermeier 1996; Buttlar 2019b).

Inhaltlich präsentiert der Wörlitzer Garten Facetten einer didaktisch-moralischen Lebensreise mit »einiger Rücksicht auf das Leben des Fürsten selbst« (Rode 1814, 102), beginnend mit dem Labyrinth und diversen Scheidewegen. Auch die von Rode als Prüfungsweg beschriebene »mystische Partie «, die dem uralten Tabula-Cebetis-Schema einer Pilgerreise mit anschließender Belohnung auf dem Berg folgt (Schleier 1973), verkörpert den Weg der »Adepten geheimer Weisheit« durch einen Parcours, der über eine gefährliche Kettenbrücke zur Einsiedlergrotte, von dort zum Betstuhl des Eremiten in felsiger Einöde und schließlich - nach weiteren Prüfungsstationen - hinauf zum Venustempel führt, einen auf mystisch-erotische Vorlagen der Antike zurückgehenden Erlösungstopos sittlicher Läuterung (Rode 1814, 125-132; Niedermeier 2007a). Der als Toleranzblick bekannte, zwischen 1779 und 1810 ausgestaltete Fächerblick, der vom Kindergrab der Goldenen Urne auf den ab 1782 als Synagoge genutzten Vestatempel, den neugotisch überhöhten Turm der Wörlitzer Kirche und auf einen Altar zuführt, der erstmals der Natur als gleichsam religiö- 


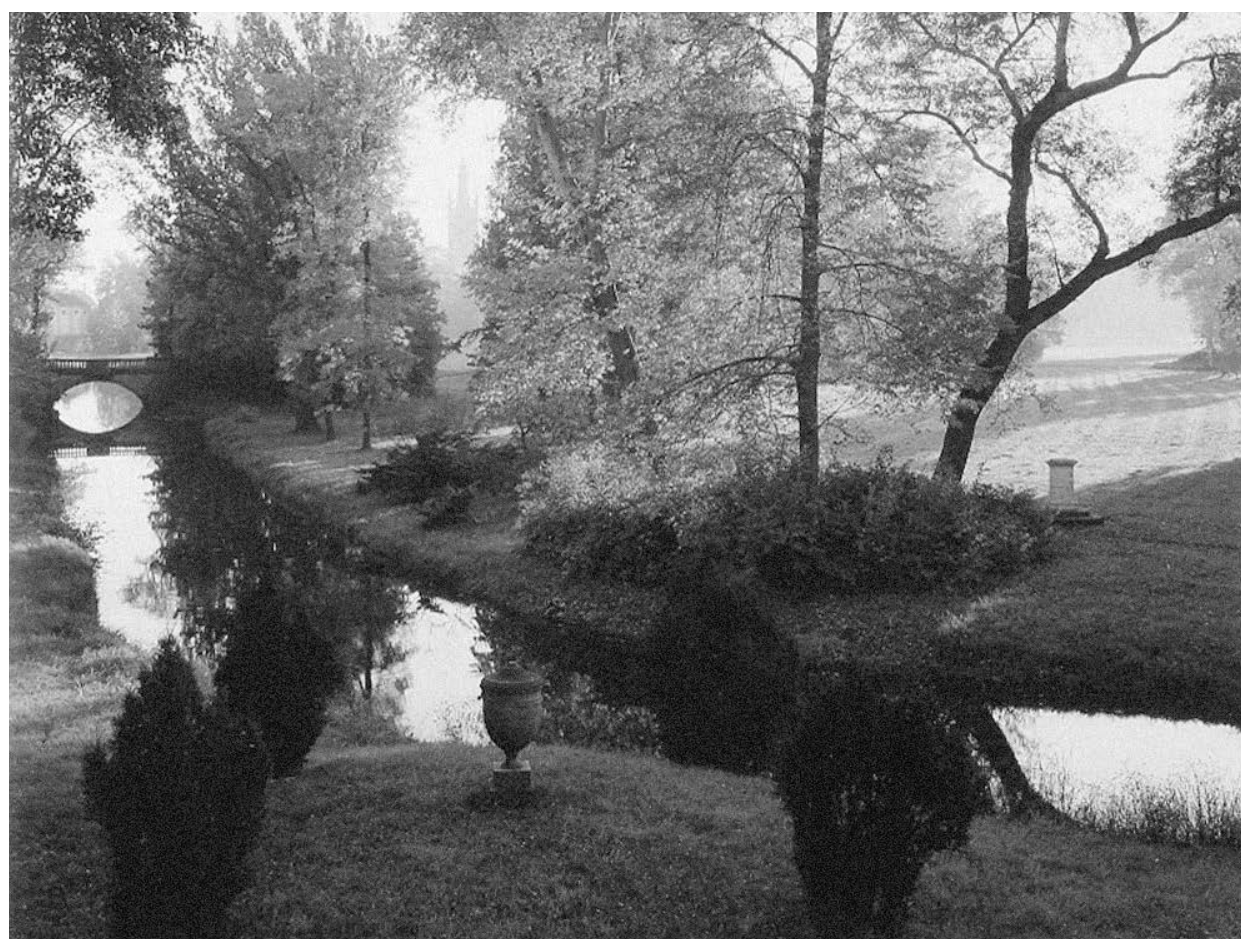

4 Wörlitz, Sichtenfächer (sog. Toleranzblick), ca. 1782/1800.

ser Instanz und ihrem Schutz gewidmet ist, belegt die These, dass Sichtachsen im Landschaftsgarten auch »Sinnachsen« sind (Günter 1980): In der Trias der religiösen Bekenntnisse (Judentum, Christentum, naturreligiöser Deismus) manifestiert sich der aufgeklärte Toleranzgedanke (Buttlar 1995, 104; Hirsch 1995, 214; Sühnel 1996, 74) (Abb. 4). Unter dem Einfluss des Archäologen Johann Joachim Winckelmann, der den Fürsten 1765 in Rom geführt hatte, entstand später ein verwandtes Narrativ am Pantheon, dessen Hypogeion den mysteriösen ägyptischen Gottheiten geweiht ist, während in der lichten antikischen Rotunde darüber die anthropomorphen Götter und Künste Griechenlands und Roms (Apoll und die Musen) verehrt werden. Dieser Aufstieg entspricht der Abfolge der Kulturstufen und hat eine Analogie im Giebelrelief, wo der Sieg der Musen über die Sirenen dargestellt ist: der Triumph der gesitteten über die wilde Triebnatur (Rode 1814, 152; Niedermeier 1995, 218-221; Tietze 1996). Schlussendlich kann auch das Wörlitzer Brückenprogramm, das vom rohen Einbaum bis zum Nachbau des modernsten zeitgenössischen Technikwunders, der Eisenbrücke von Coalbrookdale (1779), reicht, als Entwicklungsgeschichte der menschlichen Zivilisation gelesen werden (Burkhardt 1996). Es war unter den Zeitgenossen unbestritten, wenngleich später lange Zeit vergessen, dass das Wörlitzer Gartenreich ein überaus modernes Programm der Aufklärung und des Humanismus verkörperte, das der Fürst 
in seinem kleinen Musterstaat nicht nur darzustellen, sondern durch seine umfassenden Reformen auch de facto zu verwirklichen suchte. Folgerichtig war der Schlosspark nie durch sichtbare oder unsichtbare Zäune verschlossen, sondern stand allen Bewohnern und Gästen stets offen.

Der Große Tiergarten zu Berlin: Mit Wörlitz teilt der Tiergarten seit dem späten 18. Jahrhundert die Funktion eines öffentlichen Gartens. Das ursprüngliche, unter Friedrich II. gärtnerisch ausgestaltete Jagdrevier zog schon im 18. Jahrhundertein großstädtisches Massenpublikum an (Hirschfeld 1779-1785, Bd. V, 72). Der erste im Zuge der Aufklärung angelegte Volkspark war der Englische Garten in München, ab 1789 im landschaftlichen Gartenstil gestaltet und Vorbild zahlreicher im 19. Jahrhundert geschaffener Stadtparks im In- und Ausland, bis hin zum New Yorker Central Park (Buttlar 1983; Kirchner 2002). Der Volkspark als eine der »allernöthigsten Kunstanstalten einer humanen und weisen Regierung « (Sckell 1825, 198) sollte durch Ausdehnung, Wegeführung und entsprechende Flächennutzungen sowohl Möglichkeiten zur körperlichen und sportlichen Ertüchtigung durch Wandern, Spazieren und Reiten als auch - über seine idealschöne Naturästhetik und die eingestreuten Denkmäler - Erbauung und Belehrung für Geist und Seele bieten. ${ }^{9}$ Nicht zuletzt diente er am Vorabend der Französischen Revolution gezielt dem Abbau der Klassenschranken, nämlich der "Annäherung aller Stände, die sich hier im Schooße der schönen Natur begegnen $«,{ }^{10}$ wozu auch der berühmte Biergarten am Chinesischen Turm in München oder der populäre Ausschank bei den Zelten am Tiergartenrand erheblich beitrugen.

Anders als der über Jahrzehnte unter der Regie eines einzigen Bauherrn und seiner Künstler gewachsene Wörlitzer Garten weist der Tiergarten mehrere abrupte Überformungen und deutlich unterscheidbare Denkmalschichten auf (Günter 1985, 137-144; Wendland/Wörner 1986; Wendland 1993; Krosigk 2001): Das barocke Rückgrat bilden die im Zuge der Reichshauptstadtplanung Albert Speers 1939 noch einmal verbreiterte Ost-WestAchse zwischen dem Brandenburger und dem (wilhelminischen) Charlottenburger Tor, heute Straße des 17. Juni, ferner der Große Stern mit seinen acht ausstrahlenden Achsen (seit 1940 ausgeweitet und aufgewertet durch die vom ehemaligen Königsplatz/Reichstag translozierte Siegessäule) sowie die Achsenfächer am Brandenburger Tor und bei den ehemaligen Zelten am nördlichen Parkrand (wo jede Allee mit anderen Baumarten bepflanzt und entsprechend benannt war). Ein Relikt der höfischen barocken Gartentradition stellt auch das von Georg Wenzeslaus von Knobelsdorff im Sinne eines Bosketts um 1750 angelegte Venusbassin (später Fischteich - rekonstruiert 2009) dar. Gegen Ende des 18. Jahrhunderts wurde dann im Südosten der Bereich der Neuen Anlagen durch Johann Ehrenreich Sello als erste landschaftliche Partie im sentimentalen frühklassischen Stil angelegt: Die zwischen den barocken Alleen und Kompartimentgrenzen gelegenen Wald- und Gebüsch-

9 Aktivnutzungen wie Kinder- und Gruppenspiele, Planschen, Joggen und Sonnenbaden, Radeln und Picknicken kamen erst mit den sozialreformerischen Bewegungen des 20. Jahrhunderts auf. Vgl. den Beitrag von M. Norton Wise in diesem Band.

10 Sckell 1825, 198; ähnlich schon Hirschfeld 1779-1785, Bd. V, 68f. 


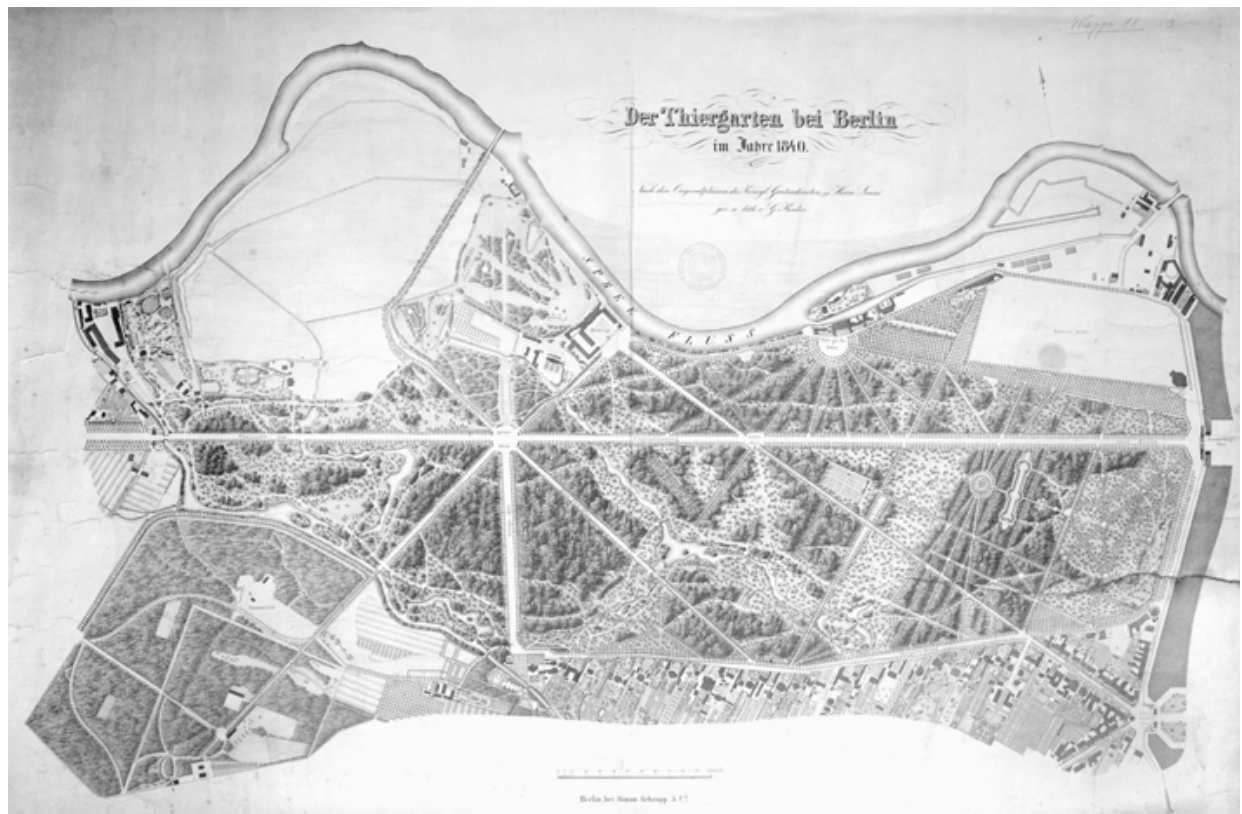

5 Der von Lenné überformte Berliner Tiergarten, Zustand 1840. „Der Thiergarten bei Berlin im Jahre 1840. Nach den Originalplänen des Königl. Gartendirektors pp. Herrn Lenné. Gez. u. lith. v. G. Koeber«.

szenen wurden nun naturalisiert, kleinteilige Bachläufe, Schlängelwege, Teiche und Inseln geschaffen und mit Denkmälern, Brücken und Statuen ausstaffiert (Rousseauinsel 1782, Luiseninsel noch zu Lebzeiten der Königin 1808). Auch sie sind heute dank jüngster Wiederherstellungen gut identifizierbar.

Die folgenreichste zweite Überformung leisteten nach Vorplanungen um 1819 von etwa 1832 bis 1840 der berühmte Potsdamer Hofgärtner und spätere Preußische Generalgartendirektor Peter Joseph Lenné (1789-1866) und seine Mitarbeiter. Er verschmolz in seinem (nur teil- und etappenweise ausgeführten) Verschönerungsplan von 1832 das barocke Rückgrat mit seiner neuen freien Gestaltung, die sich zunächst einmal durch das Ausholzen und Auflichten des dichten Waldbestandes auszeichnete, wie der Vergleich des Zustandsplanes von 1833 mit der Ausführung um 1840 belegt (Abb. 5). ${ }^{11}$ Das noch starre kleinteilige Wegenetz mit unzähligen kleinen Sternplätzen wurde aufgegeben zugunsten breiter, natürlich wirkender und im Bodenprofil gelegentlich sanft modellierter Sichtschneisen sowie einiger

11 Peter Joseph Lenné: Verschoenerungs Plan vom Königl: Thiergarten bei Berlin 1832 (Berlin Staatl. Schlösser und Gärten, Volkspark und Arkadien Nr. 153) - Situationsplan des Thier-Gartens bei Berlin 1833 [https://upload.wikimedia.org/wikipedia/de/1/1e/Tiergarten_1833.jpg] - Der Thiergarten bei Berlin im Jahre 1840 Nach den Originalplänen des Königl. Gartendirectors pp Herrn Lenné gez. und lith. von G. Koeber (Günther 1985, 140f.), vgl. Günther/Harksen 1984, 12-29. 
weniger, in entspannten elliptischen Kurven verlaufender Hauptwege (deren Form auf den französischen Gartenkünstler Gabriel Thouin zurückzuführen ist). Diese Wege waren - im Gegensatz zu den teilweise beibehaltenen barocken Achsen - nicht mehr von symmetrisch gesetzten Alleebäumen flankiert, sondern von Solitären, Baumgruppen (clumps) und Gebüschen (shrubbery). Dazwischen liegen die als Lichtspender wirkenden, erheblich vergrößerten Wasser- bzw. Teichflächen. Die sorgsame, auf Kontur der Baumkronen sowie Staffelung und Farbklang berechnete Mischung der quantitativ überwiegenden Laubbäume schließt sich zu malerischen, räumlichen Parkbildern zusammen. Von Sckells Überformung des barocken Nymphenburger Schlossparks ab 1808 lernte Lenné, dass die Beibehaltung und moderate Naturalisierung barocker Achsen und Elemente keine Verlegenheitslösung war, sondern zu einem eigenständigen »gemischten Stil« führen konnte, wie er tatsächlich um die Mitte des 19. Jahrhunderts zum Markenzeichen der Lenné-Meyer'schen-Schule wurde (Meyer 1860; Hennebo 1989).

Seine dritte Überformung erlebte der Große Tiergarten in der wilhelminischen Zeit ab den 1880er Jahren, als der sogenannte Neubarock zum Lieblingsstil des Kaisers avancierte. Die formalen Alleen und geometrischen Schmuckplätze wurden nun wieder stärker betont bzw. neu angelegt und mit pompösen Denkmalprogrammen ausgestattet (etwa die vom Kaiser geschenkte »Siegesallee« 1895-1901, deren 41 Denkmäler allen regierenden Herrschern Brandenburgs und Preußens vom Mittelalter bis in die Gegenwart gewidmet waren - die Relikte heute in der Zitadelle Spandau). Im Zuge der vierten Überformung ab den späten 1940er Jahren, als man den durch Abholzung zerstörten Tiergarten als Anbaufläche für Kartoffeln und Gemüse nutzte und nun völlig neu aufpflanzen musste (von 200.000 Bäumen hatten nur etwa 700 überlebt), entstand unter Leitung von Wilhelm Alverdes nicht nur eine - heute prächtig ausgewachsene - annähernde Rekonstruktion des Vorkriegszustandes (bei weitgehender Eliminierung der barocken bzw. wilhelminischen Denkmalschicht - markante Teilrekonstruktionen nach Vorschlägen Klaus von Krosigks seit 1990), sondern ein Volkspark, der auch ganz neue Partien einschloss, wie den 1952 aus britischen Spenden angelegten und nach dem britischen Außenminister benannten »Garten Eden « am Englischen Teehaus oder die 1957 von Gartenarchitekten wie u.a. Hertha Hammerbacher, Walter Rossow und Hermann Mattern konzipierten, in das Interbauprojekt integrierten Grünflächen des Hansaviertels (Schulz/Lingenauber 2007).

Babelsberg und Branitz: Vergleichen wir unsere Mustergärten Babelsberg und Branitz, so scheinen sie auf den ersten Blick in die gleiche Kategorie zu gehören. Beide Anlagen sind als romantische Schlossparks um die Mitte des 19. Jahrhunderts entstanden, im Resultat geprägt durch einen neu aufgepflanzten reichen einheimischen Baumbestand, prachtvolle Parkbilder und Blickachsen sowie einen raffinierten, das jeweilige Schloss umspielenden Pleasureground nach dem Entwurf des damals originellsten deutschen Landschaftsgärtners: Hermann Fürst von Pückler-Muskau. Babelsberg war 1834 für Prinz Wilhelm von Preußen (den nachmaligen ersten Kaiser) und seine Gemahlin Augusta nach dem Entwurf Karl Friedrich Schinkels und Peter Joseph Lennés als weiterer Baustein des Lenné'schen Parkgürtels seines Verschönerungsplanes der Umgebung von Potsdam (1833) begonnen 
worden. ${ }^{12}$ Kronprinz Friedrich Wilhelm (IV.) hatte diese Idee explizit von Wörlitz übernommen, als er feststellte: »Der Herzog von Dessau hat aus seinem Lande einen großen Garten gemacht, das kann ich ihm nicht nachmachen, dazu ist mein Land zu groß. Aber aus der Umgebung von Berlin und Potsdam könnte ich nach und nach einen Garten machen « (zit. n. Dehio 1961, 87). Die Grundplanung des bergigen, nach Abholzung des alten Kiefernund Eichenbestandes um 1807 fast kahlen Geländes geht auf Lenné zurück. Pückler kritisierte 1842 dessen Ansatz in »fruchtbringender Konkurrenz« (Seiler 2016) in einem Promemoria (Rippl 1989, 123-132) und überformte ab 1843 die mittlerweile aus Tausenden von Laubbäumen aufgepflanzte klassische Parklandschaft, indem er Freiräume, Kulissenpflanzungen, Blickachsen und Wegeführung ausdifferenzierte und den Park insbesondere durch Wasserflächen und Wasserläufe belebte (Bau des Dampfmaschinenhauses und des Reservoirs Schwarzes Meer auf der Anhöhe sowie einiger Wasserstürze und Bäche). Er schmückte die Terrassen- und Separatgärten des artifiziellen Pleasuregrounds farbenfroh und luxuriös aus, die den Empfehlungen Humphry Reptons folgend die nähere Umgebung des neugotischen Schlosses wohnlich und zugleich repräsentativ erscheinen lassen (Rippl 1989, 106-132; Schröder 2005; 2016). Eine besondere Verschmelzung von Tradition und realistischer Naturnachahmung gelang ihm mit der großen Fontäne in der Havel, die wie einst in den Barockgärten von Macht und Anspruch des Fürsten kündet, zugleich aber einen nordischen Geysir darstellt. ${ }^{13}$ Die nordische Anmutung Babelsbergs - sowohl im Landschaftscharakter als auch in dem auf das Mittelalter bezogenen Baustil des Schlosses und der verstreuten Nebenbauten - steht in gewolltem Kontrast zur Italianità des gegenüberliegenden Parks Glienicke von Wilhelms jüngerem Bruder Carl. Vergleicht man Babelsberg mit Branitz (1847 bis nach 1871), dem Alterssitz des Fürsten Pückler (Rippl 1989, 132-176; Streidt/Wecke 2016), so sind zwar ähnliche Stilmittel in der Zonierung, Pflanzung und Modellierung erkennbar, doch entsteht ein völlig anderes Stimmungsbild: Die ursprünglich flache, sandige und monotone Topographie, die sich zwischen dem überformten Schlösschen aus dem 18. Jahrhundert und den ab 1856 am Südende aufgeschütteten Pyramiden (Grablege des Fürsten und seiner Gattin) erstreckte, hat Pückler überaus kunstvoll durch Binnenräume, (55.000 Quadratmeter ausgehobene) Wasserläufe und Seen, abwechslungsreiche Durchblicke und (bis auf 18 Meter aufsteigende) künstliche Hügel, majestätische Baummassen und charaktervolle Solitäre in raffinierten Farbabstufungen gestaltet. Im Ergebnis ist dies - und darin liegt Pücklers besondere Kunst (Abb. 6) - eher ein dynamisches Raumkunstwerk als eine starre, auf wenige Betrachterstandpunkte berechnete Vedutenfolge. Pückler hatte - nach dem Verkauf seines Muskauer "Gartenreiches « 1846 - den alten Familiensitz Branitz als »Oasis in einer Wüste« nur durch extensive Terrainmodulationen, Anpflanzung von mehr als 100.000 Gehölzen und unter Ausnutzung

12 Verschönerungsplan der Umgebung von Potsdam 1833 entworfen von Lenné (Günther 1985, 84f.); Plansammlung der SPSG Potsdam.

13 M. Norton Wise $(2016,205)$ zweifelt allerdings daran, »dass eine solche Prahlerei auf Pücklers Einfluss zurückging - die Ästhetik seiner Landschaftsgestaltung beruhte auf Subtilität«. 
6 Illustrationstafel III »Gezwungen gepflanzte Baumgruppe - Natürlich gepflanzte Baumgruppe«. In: Fürst von Pückler-Muskau, Hermann (1834): Andeutungen über Landschaftgärtnerei. Stuttgart: Halberger.

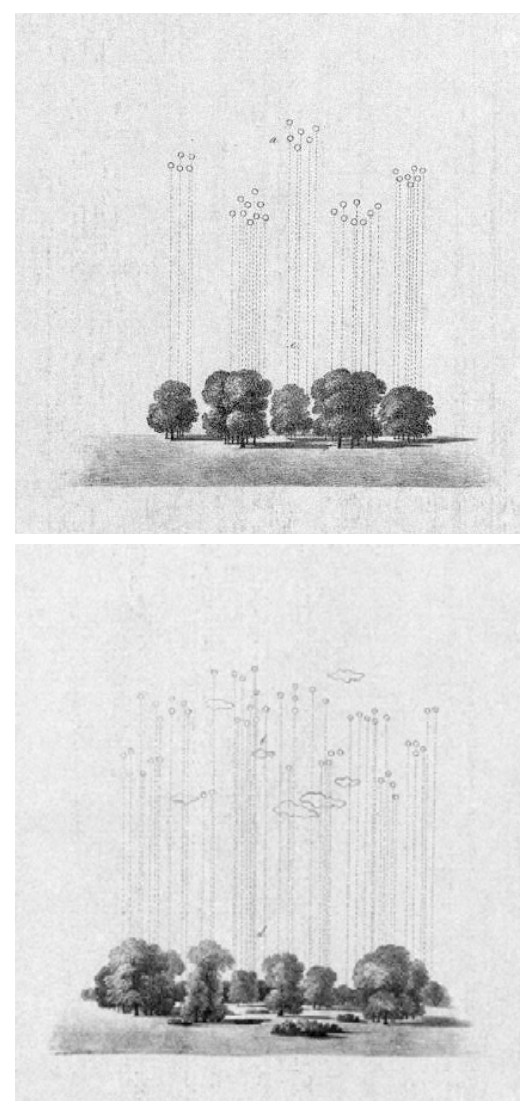

des knapp einen Meter unter Bodenniveau liegenden Grundwasserspiegels schaffen können (Potente 1906, 58-61). Bis ins Einzelne plante er die Umsetzung der Farbenlehre seines Chefgärtners Eduard Petzold, etwa an der Kulisse eines Kiefernhochwalds, der er »[...] nicht nur eine sehr malerische gezackte Linie gegen den Himmel, sondern auch ganz verschiedene Farben gegeben [habe], indem die vorderen Gruppen schwarzgrün hervortreten, die entfernteren lichtgrün erscheinen, und die ganz weiten, die nun erst sichtbar geworden, in verschiedenen blauen Nuancen sich darstellen, eine ganz künstlerische Nuancierung « (Pückler an Petzold, 6. Januar 1853, in: Stiftung Fürst Pückler Museum, Park und Schloß Branitz 1997, 37). Pücklers erweiterter Kunstbegriff, der unter dem Signum der Poesie alle Facetten der menschlichen Existenz: Ästhetik, Wissenschaft, Gesellschaft, Wirtschaft und Religion umgreife, führe trotz immer neuer Sinnangebote seiner Interpreten letztlich zu einem deutungsoffenen Kunstwerk (Jacob 2016, 37-39), das 150 Jahre nach seiner Vollendung heute noch (oder wieder) in schönster Blüte steht. 


\section{Schlussfolgerungen}

Anders als Branitz und das Wörlitzer Gartenreich, die als »Zierde und Inbegriff des 18. Jahrhunderts« (Hirsch 1985) bzw. als Höhepunkt der Pückler'schen Landschaftskunst erlebt werden sollen und entsprechend fokussiert denkmalpflegerisch restauriert wurden, liegt die Bedeutung des Berliner Tiergartens stärker in seiner historischen Entwicklung und der damit verbundenen künstlerischen Verschmelzung verschiedener Denkmalschichten. Das heißt aber nicht, dass sich dieser so beliebte Volkspark immerfort weiter transformieren lässt. Seit 1991 ist der Tiergarten als Gartendenkmal eingetragen: Seine Entwicklung und rekonstruktive Gestaltung ist mit der jüngsten Implementierung der Gedenkstätten im Nordostbereich abgeschlossen und bedarf fortan pflegender Konservierung, was weiteren Anpassungen an extensive Nutzungswünsche engste Grenzen setzen sollte. Das trifft bis zu einem gewissen Grade auch für Babelsberg zu, dessen hoher Kunstwert trotz der außerordentlichen Erhaltungsbemühungen der zuständigen Gärtner durch den brutalen Bau der DDR-Grenzanlagen stark gestört wurde und in der Öffentlichkeit lange vergessen war, zumal sich hier schon nach dem Ende der Monarchie 1918 gegen das vermeintliche Monument des Feudalismus ein Anspruch auf eine volksparkähnliche Nutzung etabliert hatte. Erst in den letzten Jahren konnte der im Zuge der Sanierung, Restaurierung und Rekonstruktion als hochrangiges Gartenkunstwerk auf denkmalverträgliche Dimensionen zurückgedrängt werden (Dorgerloh 2016).

Der vergleichende Blick zeigt, dass es nicht nur hinsichtlich der sich verändernden Nutzungsanforderungen, sondern auch der potentiellen Bedrohungen und Herausforderungen, die der Klimawandel mit sich bringt, für jedes Werk der Garten- und Landschaftskunst eine individuelle Ermittlung seines künstlerischen und (kunst)historischen Wertes geben muss. Nur auf dieser Basis lassen sich dann im Rahmen interdisziplinärer Planungen entsprechende konservatorische und rekonstruierende Maßnahmen definieren, die auch neuen Anforderungen an den Garten als Ökotop und Soziotop Rechnung tragen (Naturschutz und Denkmalpflege 1998).

\section{Literaturverzeichnis}

Addison, Joseph (1712): On the Pleasures of the Imagination. In: Spectator 411-421. (Nachdruck 1812 in: British Classics, Bd. 4. London, 71-132).

Alison, Archibald (1790): Essays on the Nature and Principles of Taste. London/Edinburgh (Nachdruck 1968. Hildesheim: Olms).

Bechtholdt, Frank-Andreas; Weiss, Thomas (Hg.): Weltbild Wörlitz - Entwurf einer Kulturlandschaft. Ostfildern-Ruit: Hatje.

Brix, Michael (2004): Der barocke Garten. André le Nôtre in Vaux-le-Vicomte. Stuttgart: Arnold.

Brix, Michael (2009): Der absolute Garten. André Le Nôtre in Versailles. Stuttgart: Arnold.

Burgsdorff, Friedrich Ludwig August von (1791): Anleitung zur sichern Erziehung und zweckmäßigen Anpflanzung der einheimischen und fremden Holzarten welche in Deutschland und unter ähnlichen Klima im Freien fortkommen. Berlin: Selbstverlag. 
Burke, Edmund (1757/1759²): A Philosophical Enquiry into the Origin of our Ideas of the Sublime and Beautiful. London: printed for R. and J. Dodsley (Reprint 1970. Menston: The Scolar Press Ltd.).

Burkhardt, Berthold (1996): Das Brückenprogramm in Wörlitz. In: Bechthold/Weiss (Hg.) (1996), 207-218.

Buttlar, Adrian von (1981): Englische Gärten in Deutschland. Bemerkungen zu Modifikationen ihrer Ikonologie. In: Sind Briten hier? Relations between British and Continental Art 1680-1880. Hg. vom Zentralinstitut für Kunstgeschichte. München: Fink, 97-126.

Buttlar, Adrian von (1982): Der Englische Landsitz 1715-1760. Symbol eines liberalen Weltentwurfs. Mittenwald: Mäander Kunstverlag.

Buttlar, Adrian von (1983): Vom Landschaftsgarten zum Volkspark. Der Englische Garten in München. In: Viktorianisches England in deutscher Perspektive. Hg. von Adolf M. Birke und Kurt Kluxen. München et al.: Saur, 133-145.

Buttlar, Adrian von (1989): Der Landschaftsgarten. Gartenkunst des Klassizismus und der Romantik Köln: DuMont.

Buttlar, Adrian von (1990): Gedanken zur Bildproblematik und zum Realitätscharakter des Landschaftsgartens. In: Die Gartenkunst 2.1, 7-17.

Buttlar, Adrian von (1995): Das Grab im Garten - Zur naturreligiösen Deutung eines arkadischen Gartenmotivs. In: Gärten und Landschaften im achtzehnten Jahrhundert. Hg. von Heinke Wunderlich. Heidelberg: Winter, 79-119.

Buttlar, Adrian von (2001): Das »Nationale« als Thema der Gartenkunst des 18. und frühen 19. Jahrhunderts [1994]. Nachdruck in: Gartenkultur und Nationale Identität - Strategien nationaler und regionaler Identitätsstiftung in der deutschen Gartenkultur. Hg. von Gert Gröning und Uwe Schneider. Worms: Werner, 21-34.

Buttlar, Adrian von (2003): Der historische Garten als Gegenstand wissenschaftlicher Forschung im 20. Jh. In: Historische Gärten - Eine Standortbestimmung. Hg. von der Vereinigung der Landesdenkmalpfleger in der Bundesrepublik Deutschland und dem Landesdenkmalamt Berlin. Berlin: Schelzky \& Jeep, 11-16.

Buttlar, Adrian von (2004): On the Limitations and Opportunities of Garden Research from the Point of View of Garden History - A Plea for Interdisciplinary Course of Study for Graduates. In: Historic Gardens today. Hg. von Michael Rhode und Rainer Schomann. Leipzig: Seemann Henschel, 104-108.

Buttlar, Adrian von (2007): »Legt Ihr's nicht aus, so legt was unter (Goethe) - On the imperative and aporia of iconological garden research. In: Prussian Gardens in Europe - 300 years of Garden History. Hg. von der Stiftung Preußische Schlösser und Gärten Berlin-Brandenburg in Zusammenarbeit mit ICOMOS/IFLA. Leipzig: Edition Leipzig, 138-141.

Buttlar, Adrian von (2008): Wer, wie, was, warum? Kritisches Einmaleins des Rekonstruierens. In: Rekonstruktion und Gartenpflege. Hg. von der Vereinigung der Landesdenkmalpfleger in der Bundesrepublik Deutschland zusammen mit Regierungspräsidium Stuttgart, Landesamt für Denkmalpflege. Imhof Verlag, 21-29.

Buttlar, Adrian von (2012): Rekonstruktionen in der Gartendenkmalpflege. In: Denkmalpflege in Bremen Unterwegs in Zwischenräumen Stadt - Garten - Denkmalpflege. Hg. von Georg Skalecki. Bremen: Edition Temmen, 331-340.

Buttlar, Adrian von (2019a): Szenen der Trauer und Melancholie im Landschaftsgarten. Beitrag zur Tagung Jardin et mélancolie, Université de Caen Normandie, 1.-2. Juni 2017, erscheint 2019 als Online-Publikation.

Buttlar, Adrian von (2019b, im Druck): Dessau-Wörlitz als Wendepunkt europäischer Gartenkunstgeschichte (Eröffnungsvortrag des Kolloquiums Leopold III. Friedrich Franz - sein Gesamtkunstwerk nach 250 Jahren, veranstaltet von der Stadt Dessau, der Kulturstiftung Dessau-Wörlitz und dem Landesamt für Denkmalpflege und Archäologie Sachsen-Anhalt, am 23. Juni 2017 in Wörlitz). Hg. von den Veranstaltern: von der Stadt Dessau, der Kulturstiftung Dessau-Wörlitz und dem Landesamt für Denkmalpflege und Archäologie Sachsen-Anhalt.

Buttlar, Adrian von; Köhler, Marcus (2012): Tod, Glück und Ruhm in Sanssouci. Ein Führer durch die Gartenwelt Friedrichs des Großen. Hg. von der Stiftung Preußische Schlösser und Gärten Berlin-Brandenburg. Ostfildern: Hatje Cantz.

Chambers, William (1772): A Dissertation on Oriental Gardening. London: W. Griffin (franz. London 1772, dt. Gotha 1775).

Dehio, Ludwig: Friedrich Wilhelm IV. von Preußen ein Baukünstler der Romantik. Deutscher Kunstverlag München/Berlin 1961. 
De Jong, Erik (1998): Der Garten als dritte Natur - Über die Verbindung von Natur und Kunst. In: Ingo Kowarik et al. (Hg.) (1998), 17-27.

Dennerlein, Ingrid (1981): Die Gartenkunst der Régence und des Rokoko in Frankreich. Worms: Werner'sche Verlagsgesellschaft.

Dezallier d'Argenville, Antoine Joseph (1760): La Théorie et la Pratique du Jardinage. Paris: C.-A. Jombert (Reprografischer Nachdruck 1972. Hildesheim/New York: Georg Olms Verlag).

Dorgerloh, Hartmut (2016): Pücklers Erbe in Babelsberg. Bewahrung und Wiederherstellung im Unesco Welterbe. In: Kunst- und Ausstellungshalle der Bundesrepublik Deutschland (Hg.) (2016), 187-191.

Erichsen-Firle, Ursula (1971): Geometrische Kompositionsprinzipien in den Theorien der Gartenkunst des 16.18. Jahrhunderts. Dissertation, Universität Köln.

Fibich, Peter (2013): Gartendenkmalpflege der DDR, Handlungsstrukturen und Positionen eines Fachgebietes. München: AVM.

Finckenstaedt, Thomas (1966): Der Garten des Königs. In: Wandlungen des Paradiesischen und Utopischen Studien zum Bild eines Ideals. Hg. von Hermann Bauer, Lorenz Dittmann, Friedrich Piel, Mohammed Rassem und Bernhard Rupprecht. Berlin: Walter de Gruyter \& Co., 183-209.

Fitzner, Sebastian (2012): Die Gartenkunst als Kunstwerk und Gattung. Über den Wandel des Kunstwerkcharakters und die Terminologie eines sich verändernden Gegenstandes. In: Schweizer/Winter (Hg.) (2012), 72-87.

Foucault, Michel (1967): Andere Räume. Nachdruck in: Aisthesis: Wahrnehmung heute oder Perspektiven einer anderen Ästhetik - Essais. Hg. von Karlheinz Barck (1993): Leipzig: Philipp Reclam, 34-46.

Gamper, Michael (1998): Die Natur ist republikanisch - Zu den ästhetischen, anthropologischen und politischen Konzepten der deutschen Gartenliteratur im 18. Jahrhundert. Würzburg: Königshausen \& Neumann.

Gerndt, Siegmar (1981): Die literarische Kontroverse um den Landschaftsgarten des 18. und frühen 19. Jahrhunderts in Deutschland. Stuttgart: Metzler.

Gilpin, William (1786): Observations - relative chiefly to picturesque Beauty. London: R. Blamire (Reprint 1973. Richmond: Richmond Publishing).

Gilpin, William (1792): Three Essays on Picturesque Beauty. London: R. Blamire (dt. Leipzig 1800).

Girardin , René-Louis Comte de (1777): De la Composition des Paysages sur le Terrain ou des Moyens d'embellir la Nature autour des Habitations En y joignant l'Agréable à l'Utile. Genf/Paris (Nachdruck Champ Vallon Editions, Ceyzerieu 1992).

Gothein, Marie Luise (1914): Geschichte der Gartenkunst. Herausgegeben mit Unterstützung der Königlichen Akademie des Bauwesens in Berlin. 2 Bde. Jena: Diederichs (Nachdruck der 2. Auflage 1926 als 4. Auflage 1997. München: Diederichs).

Günther, Harri (1980): »Ein solches Hilfsmittel möchte man sich bei allen weiten Aussichten wünschen« - Die Sichtachsen in den Wörlitzer Anlagen. In: Mitteilungen der Kommission zur Erforschung und Pflege des Wörlitzer Kulturkreises. Hg. von Erhard Hirsch und Thomas Höhle. Halle (Saale): Martin-Luther-Universität Halle-Wittenberg, 5-18.

Günther, Harri (1985): Peter Jospeh Lenné. Gärten, Parke, Landschaften. Stuttgart: DVA.

Günther, Harri; Harksen, Sibylle (1984): Bestandskatalog der Lennépläne in der Plankammer der Staatlichen Schlösser und Gärten Potsdam-Sanssouci, Teil II - Berlin. Potsdam: Staatliche Schlösser und Gärten Potsdam-Sanssouci.

Hallbaum, Franz (1927): Der Landschaftsgarten. Sein Entstehen und seine Einführung in Deutschland durch Friedrich Ludwig von Sckell 1750-1823. München: Schmidt.

Hennebo, Dieter (1989): Vom »klassischen Landschaftsgarten« zum »gemischten Stil« - Zeittypische Gestaltungstendenzen bei Peter Joseph Lenné. In: Peter Jospeh Lenné - Volkspark und Arkadien. Hg. im Auftrag der Senatsverwaltung für Stadtentwicklung und Umweltschutz von Florian von Buttlar. Berlin: Nicolaische Verlagsbuchhandlung, 49-59.

Hirsch, Erhard (1985): Dessau-Wörlitz. Zierde und Inbegriff des 18. Jahrhunderts. München/Leipzig: Beck.

Hirsch, Erhard (1995): Hortus oeconomicus: Nutzen Schönheit, Bildung. Das Dessau-Wörlitzer Gartenreich als Landschaftsgestaltung der europäischen Aufklärung. In: Gärten und Landschaften im achtzehnten Jahrhundert. Hg. von Heinke Wunderlich. Heidelberg: Carl Winter, 179-207.

Hirschfeld, Christian Cay Lorenz (1779-1785): Theorie der Gartenkunst. 5 Bde. Leipzig: M.G. Weidmanns Erben und Reich (Reprint 1973/1985². Hildesheim/New York: G. Olms).

Hogarth, William (1753): The Analysis of Beauty. London: John Reeves. 
Holmes, Caroline (2006): A Rose by Any Other Name? - An Introduction to the Symbolism of Plants and Planting. In: Symbolism in $18^{\text {th }}$-Century Gardens. The Influence of Intellectual and Esoteric Currents, such as Freemasonry/Symbolik in den Gärten des 18. Jahrhunderts. Der Einfluss unterschiedlicher philosophischer Strömungen, wie auch der Freimaurerei. Hg. von Jan. A. Snoek, Monika Scholl und Andréa Kroon. Den Haag: OVN, 77-106.

Hunt, John Dixon (1976): The Figure in the Landscape: Poetry, Painting, and Gardening during the Eighteenth Century. Baltimore/London: The Johns Hopkins University Press.

Hunt, John Dixon (1992): Gardens and the Picturesque: studies in the history of landscape architecture. Cambridge, Mass: MIT Press.

Hunt, John Dixon (2002): The Picturesque Garden in Europe. London: Thames \& Hudson.

Hunt, John Dixon; Willis, Peter $\left(1979^{2}\right)$ : The Genius of the Place. The English Landscape Garden 1660-1820. London: Elek.

Jacob, Ulf (2016): Hermann Fürst von Pückler-Muskau. Ein Künstlerleben. In: Kunst- und Ausstellungshalle der Bundesrepublik Deutschland (Hg.), 23-39.

Karg, Detlef (1989): Zur Methode denkmalpflegerischer Tätigkeit in landschaftlich gestalteten Parks. In: Fürst von Pückler-Muskau: Gartenkunst und Denkmalpflege. Hg. von Hermann Ludwig Heinrich. Weimar: Böhlau Verlag, 215-224.

Kehn, Wolfgang (1992): Christian Cay Lorenz Hirschfeld, 1742-1792, eine Biographie. Worms: Werner'sche Verlagsgesellschaft.

Kirchner, Franziska (2002): Der Central Park in New York und der Einfluss der deutschen Gartentheorie und -praxis auf seine Gestaltung. Worms: Werner'sche Verlagsgesellschaft.

Kowarik, Ingo; Schmitt, Erika; Sigel, Brigitt (Hg.) (1998): Naturschutz und Denkmalpflege. Wege zu einem Dia$\log$ im Garten. Zürich: Hochschulverlag AG an der ETH.

Krause, Katharina (1996): Die Maisons de plaisance - Landhäuser in der Île-de-France. Berlin/München: Deutscher Kunstverlag.

Krosigk, Klaus von (2001): Der Berliner Tiergarten. Berlin: Berlin Edition.

Kunst- und Ausstellungshalle der Bundesrepublik Deutschland (Hg.) (2016): Parkomanie. Die Gartenlandschaften des Fürsten Pückler in Muskau, Babelsberg und Branitz. München et al.: Prestel.

Landeshauptstadt Stuttgart (Hg.) (1994): Kongreßdokumentation zum Projekt »Natur im Kopf «, Stuttgart 21.-26. Juni 1993. 2 Bde. Stuttgart: fromman-holzboog.

Lang, Suzanne (1974): The Genesis of the English Landscape Garden. In: The Picturesque Garden and its Influence outside the British Isles. Hg. von Nikolaus Pevsner. Washington DC: Dumbarton Oaks, Trustees for Harvard University, 3-8.

Lauterbach, Iris (1987): Der französische Garten am Ende des Ancien Régime. "Schöne Ordnung" und "geschmackvolles Ebenmaß«. Worms: Werner'sche Verlagsgesellschaft.

Lauterbach, Iris (2016): Werdende Bilder im Übergang; Gartenkunst und Landschaftsmalerei. In: Kunst- und Ausstellungshalle der Bundesrepublik Deutschland (Hg.) (2016), 41-54.

Lott, Kirsten (1994): Der Obstbau im Gartenreich. Einführung in die Geschichte der frühen Obstpflanzungen. In: Das Gartenreich an Elbe und Mulde. Staatliche Schlösser und Gärten Wörlitz - Oranienbaum - Luisium. Murnau: Ubik-Verlag, 56-65.

Mason, William (1772/1786²): The English Garden. A poem in Four Books, A New Edition Corrected. Dublin: Printed by P. Byrne. https://archive.org/stream/englishgardenpoe00masoiala\#page/n0/mode/2up (30.10. 2018).

Meyer, Gustav (1860): Lehrbuch der schönen Gartenkunst. Berlin: Riegel (Nachdruck 1999, Berlin: Nicolaische Verlagsbuchhandlung).

Meyer, Margita M. (о. J.): Parkpflegewerke für Gartendenkmale - Leitlinien. http://www.historischegaerten.de/ Gartendenkmalpflege/Parkpflegewerk.html (30.10.2018).

Nath-Esser, Martina (1990): Historische Pflanzenverwendung in Landschaftsgärten: Auswertung für den Artenschutz. Worms: Werner'sche Verlagsgesellschaft.

Niedermeier, Michael (1995): Erotik und das Gartenreich Dessau-Wörlitz. In: Erotik in der Gartenkunst. Eine Kulturgeschichte der Liebesgärten. Leipzig: Edition Leipzig, 191-228.

Niedermeier, Michael (1996): Aufklärung im Gartenreich Dessau-Wörlitz. In: Bechthold/Weiss (Hg.) (1996), 51-65. 
Niedermeier, Michael (2007a): Von der Schrift in die Landschaft. Die Isis-Initiation des Apulejus in der Mystischen Partie des Wörlitzer Gartens. In: Übersetzung und Transformation. Hg. von Hartmut Böhme, Christof Rapp und Wolfgang Rösler. Berlin/New York: de Gruyter, 267-308.

Niedermeier, Michael (2007b): Vor- und frühgeschichtliche Grabdenkmäler im frühen Landschaftsgarten. In: Creating identities: Die Funktion von Grabmalen und öffentlichen Denkmalen in Gruppenbildungsprozessen. Hg. von Reiner Sörries und Stefanie Knöll. Kassel: Arbeitsgemeinschaft Friedhof und Denkmal, $163-172$.

Niedermeier, Michael (2012): Semantik. Ikonographische Gartenprogramme. In: Schweizer/Winter (Hg.) (2012), 327-352.

Norton-Wise, Matthew (2016): »Wasser auf dem dürren Hügel - Mit Dampfkraft und Ingenieurkunst zum blühenden Landschaftsgarten. In: Kunst- und Ausstellungshalle der Bundesrepublik Deutschland (Hg.) (2016), 201-205.

Osborn, James M. (1966): Joseph Spence. Observations, Anecdotes, and Characters of Books and Men, collected from Conversation, 2 Bde. Oxford: Oxford University Press.

Ovid (Publius Ovidius Naso) (1497/1501²): Ovidio metamorphoseos vulgare. Venedig.

Kunst- und Ausstellungshalle der Bundesrepublik Deutschland (Hg.) (2016): Parkomanie. Die Gartenlandschaften des Fürsten Pückler. München et al.: Prestel Verlag.

Pope, Alexander (1731): Epistles to several Persons IV: Epistle to Richard Boyle, Earl of Burlington. London: L. Gilliver. https://rpo.library.utoronto.ca/poems/epistles-several-persons-epistle-iv (30.10.2018).

Potente, Georg: Der Ausflug nach Kottbus und Branitz am 19. Juli 1906. In: Stiftung Fürst Pückler Museum, Park und Schloß Branitz (Hg.) (1997), 58-61.

Pückler; Hermann (1834): Andeutungen über Landschaftsgärtnerei verbunden mit der Beschreibung ihrer praktischen Anwendung in Muskau. Leipzig: J. B. Hirschfeld (Nachdruck 1988. Hg. von J. P. Vaupel. Frankfurt Main/Leipzig: Europäischer Hochschulverlag Bremen).

Repton, Humphrey (1795): Sketches and Hints on Landscape Gardening. London: Printed by W. Bulmer and Co.

Repton, Humphrey $\left(1803 / 1805^{2}\right)$ : Observations on the Theory and Practice of Landscape Gardening. London: Taylor.

Repton, Humphrey (1816): Fragments on the Theory and Practice of Landscape Gardening, London: Printed by T. Bensley \& son for J. Taylor.

Rippl, Helmut (1989): Pücklers Parkanlagen in Muskau, Babelsberg und Branitz. In: Fürst von Pückler-Muskau: Gartenkunst und Denkmalspflege. Hg. von Hermann Ludwig Heinrich. Weimar: Böhlau Verlag, 106-132.

Rode, August von (1814²): Beschreibung des Fürstlich Anhalt-Dessauischen Landhauses und Englischen Gartens zu Wörlitz. Dessau (Reprint der zweiten Ausgabe 1996, Wörlitz: Kettmann-Verlag).

Rohde, Michael (1998): Von Muskau bis Konstantinopel. Eduard Petzold - ein europäischer Gartenkünstler. 1815-1891. Dresden et al.: Verlag der Kunst.

Rohde, Michael; Schomann, Rainer (Hg.) (2003): Historische Gärten heute - Zum 80. Geburtstag von Professor Dr. Dieter Hennebo. Leipzig: Edition Leipzig.

Rommel, Alfred (1954): Die Entstehung des klassischen französischen Gartens im Spiegel der Sprache. Berlin: Akademie-Verlag.

Ruge, Berit (2013): Von der Finsternis zum Licht. Inszenierte Erkenntnisreisen in Gärten des Gold- und Rosenkreuzers Friedrich Wilhelm II. Worms: Werner'sche Verlagsgesellschaft.

Schepers, Wolfgang (1980): Hirschfelds Theorie der Gartenkunst 1779-1785. Worms: Werner'sche Verlagsgesellschaft.

Schleier, Reinhart (1973): Tabula Cebetis - oder »Spiegel des Menschlichen Lebens/darin Tugent und untugent abgemalet ist «, Studien zur Rezeption einer antiken Bildbeschreibung im 16. und 17. Jh. Berlin: Gebr. Mann Verlag.

Schröder, Katrin (2005): Potsdam Park Babelsberg. In: Peter Joseph Lenné, Parks und Gärten in Brandenburg, Werkverzeichnis. Hg. vom Brandenburgischen Landesamt für Denkmalpflege, dem Archäologischen Landesmuseum und der Stiftung Preußische Schlösser und Gärten Berlin-Brandenburg. Worms: Werner'sche Verlagsgesellschaft, 109-118.

Schröder, Katrin (2016): Der "Zauberer - Pücklers Gartendienste für das preußische Prinzenpaar Wilhelm und Augusta in Babelsberg. In: Kunst- und Ausstellungshalle der Bundesrepublik Deutschland (Hg.) (2016), 173-183. 
Schulz, Gabriele; Lingenauber, Klaus (2007): Die Freiräume und Gartenanlagen des Hansaviertels. In: Das Hansaviertel in Berlin. Bedeutung, Rezeption, Sanierung. Hg. vom Landesdenkmalamt Berlin. Petersberg: Imhof, 29-35.

Schweizer, Stefan (2013): Die Erfindung der Gartenkunst. Gattungsautonomie - Diskursgeschichte - Kunstwerkanspruch. Berlin: Deutscher Kunstverlag.

Schweizer, Stefan; Winter, Sascha (Hg.) (2012): Gartenkunst in Deutschland. Von der frühen Neuzeit bis zur Gegenwart, Geschichte - Themen - Perspektiven. Regensburg: Schnell + Steiner.

Sckell, Friedrich Ludwig (1818/1825²): Beiträge zur Bildenden Gartenkunst für angehende Gartenkünstler und Gartenliebhaber. München: Jos. Lindauer'sche Buchhandlung (Nachdruck 1982. Worms: Werner'sche Verlagsgesellschaft).

Seiler, Michael (2016): Fürst Hermann Pückler-Muskau und Generaldirektor Lenné - eine fruchtbringende Konkurrenz? In: Kunst- und Ausstellungshalle der Bundesrepublik Deutschland (Hg.) (2016), 207-217.

Shenstone, William (1764): Unconnected Thoughts on Gardening. In: Works in Prose and Verse of W. S., Bd. 2. London: Printed for J. Dodsley, 111-133.

Siegmund, Andrea (2011): Der Landschaftsgarten als Gegenwelt - Ein Beitrag zur Theorie der Landschaft im Spannungsfeld von Aufklärung, Empfindsamkeit, Romantik und Gegenaufklärung. Würzburg: Königshausen \& Neumann.

Stempel, Karin (1982): Geschichtsbilder im frühen englischen Garten. Fields of Remembrance - Gardens of Delight, 2 Bde. Münster: Lit. Verlag.

Stiftung Fürst Pückler Museum, Park und Schloß Branitz (Hg.) (1997): Im Spiegel der Erinnerung. Der Branitzer Park Gartenparadies des Fürsten Pückler - 24 Beiträge aus der Zeit von 1804-1939. Branitz: Fürst-Pückler-Museum.

Streidt, Gert; Wecke, Claudia (2016): Branitz, »die grösste der Oasen«. In: Kunst- und Ausstellungshalle der Bundesrepublik Deutschland (Hg.) (2016), 241-251.

Stritzke, Klaus (1998): Bäume als archäologische oder geschichtliche Dokumente. In: Ingo Kowarik et al. (Hg.) (1998), 229-236.

Sühnel, Rudolf (1996): Der englische Landschaftsgarten in Wörlitz als Gesamtkunstwerk der Aufklärung - Fünf historische Rundgänge. In: Bechthold/Weiss (Hg.) (1996), 67-84.

Tabarasi, Ana-Stanca (2007): Der Landschaftsgarten als Lebensmodell. Zur Bedeutung der »Gartenrevolution" in Europa. Würzburg: Könighausen \& Neumann.

Trauzettel, Ludwig (1996): Gartenkünstler und Gartenkunst in Wörlitz. In: Bechthold/Weiss (Hg.) (1996), 85-98 und Katalogteil.

Trauzettel, Ludwig (2003): Die Pflege der Wörlitzer Gärten seit 1982. In: Rohde/Schomann (Hg.) (2003), 228-233.

Trauzettel, Ludwig (2006): Die Wörlitzer Anlagen, Der Schlossgarten, Neumarks Garten, Schochs Garten, Die Neuen Anlagen, Die Stadt Wörlitz. In: Unendlich schön - Das Gartenreich Dessau-Wörlitz. Hg. von der Kulturstiftung Dessau-Wörlitz. Berlin: Nicolaische Verlagsbuchhandlung, 160-200.

Walpole, Horace (1785): Essay On modern Gardening = A History of the Modern Taste in Gardening/Essai sur l'Art des Jardins. Strawberry Hill (Reprint 1994. Canton, Pennsylvania: The Kirgate Press). https://archive. org/stream/essayonmodernga00walpgoog\#page/n8/mode/2up (30.10.2018).

Verschragen, Jeroen (2001): Die »stummen Führer« der Spaziergänger: Über die Wege im Landschaftsgarten. Frankfurt a.M.: Peter Lang.

Wecke, Claudia (2016): "Es ist die Freiheit der Bäume, nach der wir uns sehnen « - Großbaumverpflanzung und Baumdiversität à la Pückler. In: Kunst- und Ausstellungshalle der Bundesrepublik Deutschland (Hg.) (2016), 253-257.

Weiss, Thomas (1997): »'eus le bonnheur de vous accompagnez...« Friedrich Wilhelm von Erdmannsdorffs Reisenotizen des Jahres 1764. In: Den Freunden der Natur und Kunst - Das Gartenreich des Fürsten Franz von Anhalt-Dessau im Zeitalter der Aufklärung - Eine Ausstellung des Instituts für Auslandsbeziehungen und der Kulturstiftung Dessau-Wörlitz. Hg. von der Kulturstiftung Dessau-Wörlitz und dem Institut für Auslandsbeziehungen und ifa. Ostfildern-Ruit: Hatje 1997, 30-72.

Wendland, Folkwin (1993): Der Große Tiergarten in Berlin - Seine Geschichte und Entwicklung in fünf Jahrhunderten. Berlin: Gebr. Mann Verlag.

Wendland, Folkwin; Wörner, Gustav; Wörner, Rose (1986): Der Berliner Tiergarten - Vergangenheit und Zukunft. Hg. vom Senator für Stadtentwicklung und Umweltschutz. Berlin: Schelzky \& Jeep. 
Weyh, Henrike (2006): »Norden« und »Dänemark« als Bilder nationaler Selbstdarstellung im dänischen Landschaftsgarten. Frankfurt a. M.: Peter Lang.

Whately, Thomas (1770): Observations on modern Gardening Illustrated by Descriptions. London (Nachdruck 1982. New York: Garland Publishing. Dt. Leipzig 1771, franz. Paris 1771).

Wimmer, Clemens Alexander (1989): Geschichte der Gartentheorie. Darmstadt: Wissenschaftliche Buchgesellschaft.

Wimmer, Clemens Alexander (2014): Lustwald, Beet und Rosenhügel - Geschichte der Pflanzenverwendung in der Gartenkunst. Weimar: Verlag Datenbank für Geisteswissenschaften.

Wimmer, Clemens Alexander (2016): Der Gartenkünstler Peter Josph Lenné. Eine Karriere am preußischen Hof. Darmstadt: Wissenschaftliche Buchgesellschaft.

\section{Bildnachweis}

1 Fine Arts Museums of San Francisco, Gift of Osgood Hooker, https://www.famsf.org; 2 Kupferstich-Kabinett, Staatliche Kunstsammlungen Dresden, Foto: Andreas Diesend; 3 Universitätsbibliothek Heidelberg, Signatur K 6338-20, Taf. I, Seite 76; 4 Adrian von Buttlar, um 2000; 5 Lenné, Peter Joseph; Gerhard Koeber: Berlin, Tiergarten, 1840, Lithographie, SPSG, GK II (1) 3594/Stiftung Preußische Schlösser und Gärten Berlin-Brandenburg/SPSG; 6 ETH-Bibliothek Zürich, Rar 1324, http://doi.org/10.3931/e-rara-13540 